Bull. Iraq nat. Hist. Mus.

(2021) 16 (4): 509-533.

https://doi.org/10.26842/binhm.7.2021.16.4.0509

\title{
A MULTIVARIATE MORPHOMETRIC ANALYSIS OF THE GENUS LOTUS L., 1753 (FABACEAE, LOTEAE) FROM EGYPT
}

\author{
Ali Gaafar* Monier Abd El-Ghani** Azza El Hadidy** \\ and \\ Ethar Hussein *** \\ * Botany Department, Faculty of Science, New Valley University, El Kharga, Egypt. \\ ** Department of Botany and Microbiology, Faculty of Science, Cairo University, Giza, \\ Egypt. \\ *** Department of Biological and Geological Sciences, Faculty of Education, Ain Shams \\ University, Cairo, Egypt. \\ •Corresponding author: ethar_asaad@yahoo.com
}

Received Date: 20 August 2021, Accepted Date: 23 October 2021, Published Date: 20 December 2021

(c) (i)

This work is licensed under a Creative Commons Attribution 4.0 International License

\section{ABSTRACT}

This study aims at examining and confirming the patterns of phenetic relationships and the levels of variations within and among the species of Lotus L., 1753 in Egypt by using morphometric analysis techniques. We have evaluated 24 morphological characters from about 300 herbarium specimens representing 19 species of Lotus that are currently recognized. Based on numerical analyses of macromorphological characters (cluster analysis, principal coordinate analysis and principal component analysis), 19 species of Lotus were recognized from Egypt. These species were clustered in six species-specific groups: (I) Lotus halophilus Boiss. \& Spruner, L. angustissimus L., L. glinoides Delile and L. schimperi Steud. ex Boiss., (II) Lotus glaber Mill. and L. palustris Willd., (III) Lotus polyphyllos E.D. Clarke, L. creticus L. and L. cytisoides L., (IV) Lotus gebelia Vent., L. lanuginosus Vent. and L. arenarius Brot., (V) Lotus edulis L., L. tetragonolobus L. and L. conjugatus L. and (VI) Lotus ornithopodioides L., L. peregrinus L., L. arabicus L. and L. hebranicus Hochst. ex Brand.

As a result of this study, we proposed that some characters, not previously examined in detail, showed significant characters in species delimitation: pod length, seed dimensions, features of upper and lower leaflets, calyx, length of corolla, length of style, numbers of flowers and ovules.

Keywords: Cluster analysis, Morphology, Numerical taxonomy, PCA, Phenetic analysis. 


\section{A multivariate morphometric analysis}

\section{INTRODUCTION}

The genus Lotus L., 1753 (Fabaceae, Loteae) is polymorphic and includes about 150 species native to Europe, Asia, Africa, Australia and some islands of Atlantic Ocean, Pacific Ocean and Socotra archipelago in the Indian Ocean. The greatest genetic diversity for Lotus occurs in the Mediterranean Basin (Grant, 1991; Sokoloff, 1998). Based on previous studies, this genus is a taxonomically difficult genus as it includes complexes of closely related groups with similar vegetative characters (Gillett, 1958; Heyn, 1967; Kramina, 1999, 2006; Kramina and Sokoloff, 2004; Kramina et al., 2016, 2018, 2020, 2021), including seasonal polymorphisms (Heyn, 1970), and it is difficult to distinguish among the species (Ojeda et al., 2009).

Historically there has been little agreement in the taxonomic literature regarding the generic limits of Lotus and its infrageneric subdivision (Degtjareva et al., 2006). All native New World species formerly placed in Lotus are now segregated in four genera (e.g. Arambarri et al., 2005; Sokoloff and Lock, 2005; Sokoloff et al., 2007) or two distinct genera (Brouillet, 2008). In the Old World, three monotypic segregate genera are accepted: Kebirita Kramina and Sokoloff, Podolotus Royle and Pseudolotus Rech. f.; while two commonly recognized genera: Dorycnium Mill. and Tetragonolobus Scop. are placed in the synonymy of Lotus (Degtjareva et al., 2006). However, this has changed considerably with the advent of phylogenetic studies based on nrITS sequences; these have clearly shown that the New World species of Lotus are not closely related to the Old World species (Allan and Porter, 2000), and in particular Degtjareva et al. (2006) revised sectional classifications proposed by Sokoloff (1999 a, b) and Kramina and Sokoloff (2003).

Some sections appeared as non-monophyletic, including the section Lotus, which was resolved as paraphyletic since Lotus conimbricensis Brot. (Lotus sect. Erythrolotus Brand) had ITS sequence type identical to those found in Lotus subbiflorus Lag. (Lotus sect. Lotus) (Faria et al., 2012).While several works dealt with the genus Lotus in Egypt, this genus was classified into six sections: Lotus, Krokeria, Erythrolotus, Lotea, Pedrosia and Quadrifolium (Muschler, 1912; Täckholm, 1974; Boulos, 1999). El Hadidy (2003, 2004) adopted the classification of Lotus L. into three subgenera Pedrosia, Lotus and Tetragonolobus and four sections Krokeria, Loteae, Lotus and Erythrolotus based on floral characters (style and stigma), fruit characters (pod and seed), as well as vegetation characters (basal leaflets) and geographical distribution. In Egypt, the taxonomy of the genus Lotus has always been problematic which has been reflected in the number of its species (Täckholm, 1974; Boulos, 2009). Several studies have demonstrated the use of micromorphological characters to differentiate between some taxa of Fabaceae (Stenglein et al., 2003; Zorić et al., 2009; Saheed and Illoh, 2010; Albert and Sharma, 2013; El-Gazzar et al., 2013).

Different techniques of multivariate analyses were increasingly applied to resolve some difficulties that may be confronted by a morphological overlap in flowering plants (e.g. Sokal and Sneath, 1963; Gilmartin, 1967; Jensen and Eshbaugh, 1976; McNeil, 1984; Jensen et al., 1993). Numerical taxonomy uses numeric algorithms to create groups of taxonomic units based on their character states. Two basic methodologies can be included within numerical 
Gaafar et al.

analyses: phenetic and cladistic (phylogenetic); in phenetic analyses, classifications are Different techniques of multivariate analyses were increasingly applied to resolve some difficulties that may be confronted by a morphological overlap in flowering plants (e.g. Sokal and Sneath, 1963; Gilmartin, 1967; Jensen and Eshbaugh, 1976; McNeil, 1984; Jensen et al., 1993). Numerical taxonomy uses numeric algorithms to create groups of taxonomic units based on their character states. Two basic methodologies can be included within numerical analyses: phenetic and cladistic (phylogenetic); in phenetic analyses, classifications are formed based on the patterns of overall similarities, usually in exomorphology. On the other hand, cladistic (phylogenetic) analyses are based on the premise of estimating the pattern of evolutionary history (phylogeny) using shared derived characters (or synapomorphies); Morphometric techniques have long been established as valuable tools for exploring the development, population differentiation and systematics of plants (Wiens, 2000; Macleod and Forey, 2002; Jensen, 2003; Bateman and Rudall, 2006; El-Hadidy et al., 2011; Ellmouni et al., 2017).

The current study was carried out to examine and confirm the patterns of phenetic relationships and the levels of variations within and among the species of Lotus in Egypt by using morphometric analysis techniques.

\section{Plant specimens}

\section{MATERIALS AND METHODS}

Nineteen species of Lotus are used in the present study (Tab. 1). The data used for the morphometric analysis are recorded from about 300 herbarium specimens deposited in Herbarium of Cairo University (CAI), Herbarium of Agricultural Research Center (CAIM) and Assiut University Herbarium (ASTU) (acronyms sensu Thiers, 2017). Intact and wellpreserved specimens are included in the analyses (Tab. 2). Species are collected from different bioclimatic zones of Egypt to represent as much as possible the entire distribution range of the taxa, as well as the morphological variation in each species. Species identification and nomenclature are made with the aid of the floras of Egypt and adjacent countries (Zohary, 1972; Boulos, 1999; Collenette, 1999).

Table (1): Classification of the studied taxa of Lotus (Callen, 1959) (A=Annual, $\mathrm{P}=$ Perennial. Abbreviations of species are used in Diagrams 1 and 2).

\begin{tabular}{|c|l|l|c|c|c|}
\hline No. & \multicolumn{1}{|c|}{ Species } & Abbreviation & Subgenus & Section & Duration \\
\hline 1 & $\begin{array}{l}\text { Lotus arenarius } \\
\text { Brot. }\end{array}$ & L. are & Pedrosia & Pedrosia & A \\
\hline 2 & L. edulis L. & L. edu & Lotus & Krokeria & A \\
\hline 3 & $\begin{array}{l}\text { L. } \text { ornithopodioides } \\
\text { L. }\end{array}$ & L. orn & Lotus & Lotea & A \\
\hline 4 & $\begin{array}{l}\text { L. halophilus } \text { Boiss. } \\
\text { \& Spruner }\end{array}$ & L. halo & Lotus & Lotea & A \\
\hline 5 & L. peregrinus L. & L. pere & Lotus & Lotea & A \\
\hline 6 & $\begin{array}{l}\text { L. polyphyllos } \text { E.D. } \\
\text { Clarke }\end{array}$ & L. poly & Lotus & Lotea & P \\
\hline 7 & L. creticus L. & L. cret & Lotus & Lotea & P \\
\hline 8 & L. cytisoides L. & L. cyt & Lotus & Lotea & P \\
\hline
\end{tabular}


A multivariate morphometric analysis

\begin{tabular}{|c|l|l|l|l|c|}
\hline 9 & L. glaber Mill. & L. gla & Lotus & Lotus & P \\
\hline 10 & L. angustissimus L. & L. ang & Lotus & Lotus & A \\
\hline 11 & L. palustris Willd. & L. pal & Lotus & Lotus & P \\
\hline 12 & L. glinoides Delile & L. glin & Lotus & Erythrolotus & A \\
\hline 13 & $\begin{array}{l}\text { L. schimperi Steud. } \\
\text { ex Boiss. }\end{array}$ & L. schim & Lotus & Erythrolotus & A \\
\hline 14 & L. arabicus L. & L. arab & Lotus & Erythrolotus & A \\
\hline 15 & $\begin{array}{l}\text { L. hebranicus } \\
\text { Hochst. ex Brand }\end{array}$ & L. heb & Lotus & Erythrolotus & A \\
\hline 16 & L. gebelia Vent. & L. geb & Lotus & Erythrolotus & A \\
\hline 17 & $\begin{array}{l}\text { L. lanuginosus } \\
\text { Vent. }\end{array}$ & L. lan & Lotus & Erythrolotus & P \\
\hline 18 & $\begin{array}{l}\text { L. tetragonolobus } \\
\text { L. }\end{array}$ & L.tetra & Tetragonolobus & Erythrolotus & A \\
\hline 19 & L. conjugatus L. & L. conj & Tetragonolobus & Erythrolotus & A \\
\hline
\end{tabular}

\section{Characters scored for morphometric analysis}

The morphometric analysis is based on 24 quantitative continuous (17) and quantitative discrete cardinal (7) characters consisting of vegetative and reproductive structures are examined (Tab. 3). In order to avoid biased data due to variations in phenetic features, 10-15 specimens for each species are examined (Tab. 2).

For the data matrix, the quantitative cardinal characters are coded as binary/multi-state characters and the means of quantitative continuous characters are also coded as multi-state characters. Measurements in the herbarium specimens are conducted using digital calipers or a ruler. Each species is encoded as an Operational Taxonomic Unit (OUT) ( Sokal and Sneath, 1963).

Table (2): The collection data for some examined specimens of Lotus taxa.

\begin{tabular}{|c|c|c|c|c|c|}
\hline No. & Taxa & Locality & Habitat & $\begin{array}{c}\text { Collection } \\
\text { date }\end{array}$ & Collector \\
\hline 1 & L. are & $\begin{array}{c}\text { Ras el Hekma, } \\
\text { Mariut }\end{array}$ & $\begin{array}{c}\text { Sandy ground by the } \\
\text { sea }\end{array}$ & $2 / 5 / 1955$ & $\begin{array}{c}\text { M.N.El } \\
\text { Hadidi }\end{array}$ \\
\hline 2 & L. $e d u$ & Burg el Arab & $\begin{array}{c}\text { Field margin, } \\
\text { roadsides, waste } \\
\text { places, coastal sand } \\
\text { dunes, rocky \& } \\
\text { limestone slopes. }\end{array}$ & $15 / 3 / 1928$ & $\begin{array}{c}\text { V. } \\
\text { Täckholm }\end{array}$ \\
\hline 3 & L. orn & $\begin{array}{c}\text { Bahariya oasis, } \\
\text { Bawiti, El Qasr. }\end{array}$ & $\begin{array}{c}\text { Moist places by } \\
\text { springs and streams; } \\
\text { edges of cultivated } \\
\text { ground and roadsides; } \\
\text { rocky wastes }\end{array}$ & $15 / 3 / 1968$ & Gun Romee \\
\hline 4 & L. halo & $\begin{array}{c}\text { Sinai, El } \\
\text { Kharruba village }\end{array}$ & $\begin{array}{c}\text { Sandy desert wadies, } \\
\text { waste ground and } \\
\text { roadsides; limestone } \\
\text { rocks, in cultivation, } \\
\text { dunes near sea shore }\end{array}$ & $3 / 4 / 1988$ & $\begin{array}{c}\text { El Hadidi } \text { et } \\
\text { al. }\end{array}$ \\
\hline
\end{tabular}


Gaafar et al.

\begin{tabular}{|c|c|c|c|c|c|}
\hline 5 & L. pere & $\begin{array}{l}\text { Bahariya oasis, } \\
\text { Bawiti, El Qasr. }\end{array}$ & $\begin{array}{l}\text { Coastal sand dunes, } \\
\text { rocky calcareous } \\
\text { slopes; in cultivated } \\
\text { ground or by } \\
\text { roadsides }\end{array}$ & $15 / 3 / 1968$ & Gun Romee \\
\hline 6 & L. poly & Sidi Kirir & $\begin{array}{c}\text { Coastal sand dunes } \\
\text { and adjacent desert } \\
\text { plains. }\end{array}$ & 23/3/1987 & A.G. Famy \\
\hline 7 & L. cret & Rosetta & $\begin{array}{l}\text { Sand dunes and sand } \\
\text { stone cliffs by the sea }\end{array}$ & 20/4/1973 & $\begin{array}{c}\text { Ibrahim } \\
\text { Mahdi \& S. } \\
\text { Sisi } \\
\end{array}$ \\
\hline 8 & L. cyt & $\begin{array}{c}\text { El Rasool } \\
\text { Village, Mersa } \\
\text { Matruh - Salum } \\
\text { road }\end{array}$ & $\begin{array}{l}\text { Sandy desert places, } \\
\text { dunes, wadies or in } \\
\text { oolothic limestone } \\
\text { rocks, usually by the } \\
\text { sea }\end{array}$ & 2/5/1988 & A.G. Famy \\
\hline 9 & L.gla & $\begin{array}{c}\text { Cairo - } \\
\text { Alexandria desert } \\
\text { road }(\mathrm{K} 48)\end{array}$ & $\begin{array}{c}\text { moist and cultivated } \\
\text { ground, canal banks, } \\
\text { lawns }\end{array}$ & 7/3/1978 & $\begin{array}{l}\text { Merxmuïlle } \\
\quad \text { r } \text { et al. }\end{array}$ \\
\hline 10 & L. ang & Kafr Siman & Usually in humid soil & $7 / 4 / 1927$ & N.Simpson \\
\hline 11 & L. pal & $\begin{array}{c}\text { Dakhla Oasis: } \\
\text { Mutat Bir } \\
\text { Asmant el Gedid }\end{array}$ & $\begin{array}{l}\text { Near rivulets and } \\
\text { ditches, in cultivated } \\
\text { ground }\end{array}$ & $11 / 2 / 1952$ & $\begin{array}{l}\text { V. } \\
\text { Täckholm } \\
\text { \& Kassas }\end{array}$ \\
\hline 12 & L.glin & $\begin{array}{l}\text { Wadi Iseili, Suez } \\
\text { road }\end{array}$ & $\begin{array}{l}\text { Sandy desert wadies } \\
\text { and plains }\end{array}$ & $8 / 1 / 1960$ & $\begin{array}{c}\mathrm{V} . \\
\text { Täckholm et } \\
\text { al. }\end{array}$ \\
\hline 13 & L.schim & $\begin{array}{l}\text { Wadi Idib, } \\
\text { "Panicum } \\
\text { turgidum } \\
\text { community" }\end{array}$ & $\begin{array}{l}\text { Sandy wadies and } \\
\text { plains }\end{array}$ & $4 / 3 / 1967$ & $\begin{array}{l}\text { D. Oshorn } \\
\text { \& I. Helmy }\end{array}$ \\
\hline 14 & L. $a r a b$ & $\begin{array}{c}\text { El Minya, } \\
\text { Eastern side, Deir } \\
\text { Al Azzra } \\
\text { Qena }\end{array}$ & $\begin{array}{l}\text { Weed on Nile banks } \\
\text { and in field }\end{array}$ & $\begin{array}{l}\text { 2/2/1979 } \\
15 / 4 / 1977\end{array}$ & $\begin{array}{l}\text { M. Amry } \\
\text { Kosinova \& } \\
\text { Slavicova } \\
\end{array}$ \\
\hline 15 & L. heb & $\begin{array}{l}\text { Thamilat Al- } \\
\text { shifa, Red Sea } \\
\text { Coast }\end{array}$ & $\begin{array}{l}\text { Sandy coastal plains; } \\
\text { foot hills; wadies in } \\
\text { calcareous and stony } \\
\text { ground in hot desert } \\
\text { areas. }\end{array}$ & 28/11/1986 & Hobbs \\
\hline 16 & L. $g e b$ & Heliopolis, Cairo & Dry and rocky places & $\begin{array}{c}1820- \\
1826 \\
\end{array}$ & Ehrenberg \\
\hline 17 & L. lan & $\begin{array}{c}\text { Sinai: El - Arish } \\
\text { - El Hassana, } 7 \\
\text { km before El } \\
\text { Hassana } \\
\end{array}$ & $\begin{array}{l}\text { Desert plains on sandy } \\
\text { gravel; in fields }\end{array}$ & 4/4/1988 & El Garf \\
\hline 18 & L. tetra & $\begin{array}{c}\text { West Mersa } \\
\text { Matruh, wadi el- } \\
\text { Ramleh }\end{array}$ & $\begin{array}{l}\text { Fields, roadsides, } \\
\text { calcareous ground and } \\
\text { waste ground }\end{array}$ & $10 / 3 / 1965$ & $\begin{array}{c}\text { V. } \\
\text { Täckholm }\end{array}$ \\
\hline
\end{tabular}


A multivariate morphometric analysis

\begin{tabular}{|c|c|c|c|c|c|}
\hline 19 & L. conj & $\begin{array}{c}\text { Sinai, Tarfa } \\
\text { district }\end{array}$ & Fields and dry places & $7 / 5 / 1982$ & H. Barakat \\
\hline
\end{tabular}

\section{Statistical treatment of data}

For morphological diversity, simple descriptive statistics for quantitative continuous parameters are calculated for each species included in the analyses using STATISTICA software version 8.0 (Weiß, 2007). A Shapiro-Wilk statistic was used (with $p<0.05$ ) to test whether any morphometric variable deviated from a normal distribution and equality of variance (Cortinhas et al., 2015). Before further statistical tests, appropriate transformations (when required) were applied to each parameter did not follow a normal distribution. The Pearson's correlation coefficients between each character pairs are computed in order to reveal highly correlated characters and to ensure that no high correlations (> 0.90) (Španiel et al., 2017), are present that could potentially affect the results of further multivariate analyses. If the correlation coefficients for the correlated pairs of variables exceeded $r=0.90$, they are excluded from the multivariate analyses.

\section{Procedures of multivariate analyses}

In order to obtain general information about the relationships and similarities of the examined morphological traits, a cluster analysis is performed on a dataset of all the 19 OTUs using 24 characters. To assess the phenetic relationships between species (OUTs), the similarity between two OTUs is calculated on the basis of Gower's general similarity coefficient, and the dendrogram is prepared using un-weighted pair-group method with arithmetic means (UPGMA) clustering algorithms with PAST 3.25 (PAlaeontological STatistics) software package (Hammer et al., 2001). Gower distance is chosen since it can handle metric characters as well as nominal and ordinal-scaled ones (Gower, 1971).

The cophenetic correlations were then calculated between the tree matrix and the similarity matrix in order to estimate how well the dendrogram represents its corresponding pairwise distance matrix. High cophenetic correlation coefficient (more than 0.7) indicates that the hierarchic classification obtained by the clustering method is a reasonably faithful representation of the original resemblance matrix (Sokal, 1986). Based on the morphological characters, the species groups that resulted from cluster analysis are subjected to ANOVA to reveal significant differences between means of characters across the identified groups (Sokal and Rohlf, 1981) using SPSS version 16.0.

A principal coordinates analysis (PCoA) is performed on the basis of the 24 morphological characters, where it is more appropriate with mixed dataset (continuous and discrete cardinal). The distance matrix is often based on Gower's coefficient (Legendre and Legendre, 1998). The goal of PCoA is the positioning of species in a space of reduced dimensionality while preserving their distance relationships.

On the basis of 17 quantitative continuous morphological characters, a principal components analysis (PCA) is applied on the matrix of product-moment correlations, obtained from the standardized data, to provide further insight into structure in the data set. This method is well- 
Gaafar et al.

suited to revealing patterns of continuous variations in a data set (Sneath and Sokal, 1973). The PCA investigates the overall variation pattern along the first two components in order to find hypothetical variables (components) that can discriminate among groups.

Morphological characters are projected onto the eigenvectors, with a priori assignment to the groups of species obtained from the classification plotted in two dimensions for examination. Results of PCA analysis is performed using CANOCO version 4.5 for windows (Ter Braak and Šmilauer, 2003), and presented as a two-dimensional scatter plot where each point represents one taxon and an arrow for a character.

Table (3): Characters and character states used for morphological characterization of

\begin{tabular}{|c|c|c|c|c|}
\hline & Characters & Abbreviation & Character states & Coded as \\
\hline \multirow[t]{2}{*}{ Stem } & 1- Life history & $\mathrm{H}$ & Annual & 1 \\
\hline & & & Perennial & 2 \\
\hline \multirow[t]{18}{*}{ Leaf } & $\begin{array}{l}\text { 2- Shape of upper } \\
\text { leaflet }\end{array}$ & SUL & Ovate & 1 \\
\hline & & & Obovate & 2 \\
\hline & & & Lanceolate & 3 \\
\hline & & & Oblanceolate & 4 \\
\hline & $\begin{array}{l}\text { 3- Length of upper } \\
\text { leaflet }\end{array}$ & ULL & $(>15 \mathrm{~mm})$ & 1 \\
\hline & & & $(<15 \mathrm{~mm})$ & 2 \\
\hline & $\begin{array}{l}\text { 4- Width of upper } \\
\text { leaflet }\end{array}$ & ULW & $(>7 \mathrm{~mm})$ & 1 \\
\hline & & & $(<7 \mathrm{~mm})$ & 2 \\
\hline & $\begin{array}{l}\text { 5- Shape of lower } \\
\text { leaflet }\end{array}$ & SLL & Ovate & 1 \\
\hline & & & Obovate & 2 \\
\hline & & & Lanceolate & 3 \\
\hline & & & Oblanceolate & 4 \\
\hline & $\begin{array}{l}\text { 6- Length Lower } \\
\text { leaflet }\end{array}$ & LLL & $(>2-10 \mathrm{~mm})$ & 1 \\
\hline & & & $(<10 \mathrm{~mm})$ & 2 \\
\hline & 7- Width lower leaflet & LLW & $(>2-5 \mathrm{~mm})$ & 1 \\
\hline & & & $(<5 \mathrm{~mm})$ & 2 \\
\hline & 8- Length of rachis & $\mathrm{R}$ & $(>4 \mathrm{~mm})$ & 1 \\
\hline & & & $(<4 \mathrm{~mm})$ & 2 \\
\hline \multirow[t]{4}{*}{ Flower } & 9- Number & NF & $(>2)$ & 1 \\
\hline & & & $(<2)$ & 2 \\
\hline & 10- Bract length & BL & $(>6 \mathrm{~mm})$ & 1 \\
\hline & & & $(<6 \mathrm{~mm})$ & 2 \\
\hline \multirow[t]{2}{*}{ Corolla } & 11- Length & CRL & $(>10 \mathrm{~mm})$ & 1 \\
\hline & & & $(<10 \mathrm{~mm})$ & 2 \\
\hline
\end{tabular}


A multivariate morphometric analysis

\begin{tabular}{|c|c|c|c|c|}
\hline & \multirow[t]{2}{*}{ 12- Color } & \multirow[t]{2}{*}{ CRC } & Yellow & 1 \\
\hline & & & Pink & 2 \\
\hline \multirow[t]{6}{*}{ Calyx } & \multirow[t]{2}{*}{ 13- Length } & \multirow[t]{2}{*}{ CL } & $(>7 \mathrm{~mm})$ & 1 \\
\hline & & & $(<7 \mathrm{~mm})$ & 2 \\
\hline & \multirow[t]{2}{*}{ 14- Tube length } & \multirow[t]{2}{*}{$\mathrm{CTU}$} & $(>3 \mathrm{~mm})$ & 1 \\
\hline & & & $(<3 \mathrm{~mm})$ & 2 \\
\hline & \multirow[t]{2}{*}{ 15- Teeth length } & \multirow[t]{2}{*}{$\mathrm{CT}$} & $(>5 \mathrm{~mm})$ & 1 \\
\hline & & & $(<5 \mathrm{~mm})$ & 2 \\
\hline \multirow[t]{5}{*}{ Style } & \multirow[t]{3}{*}{ 16- Shape } & \multirow[t]{3}{*}{ STS } & Bifid & 1 \\
\hline & & & Simple swollen & 2 \\
\hline & & & Simple un-swollen & 3 \\
\hline & \multirow[t]{2}{*}{ 17- Length } & \multirow[t]{2}{*}{ STL } & $(>5 \mathrm{~mm})$ & 1 \\
\hline & & & $<5 \mathrm{~mm})$ & 2 \\
\hline \multirow[t]{4}{*}{ Pod } & \multirow[t]{2}{*}{ 18- Length } & \multirow[t]{2}{*}{ PL } & $(>30 \mathrm{~mm})$ & 1 \\
\hline & & & $(<30 \mathrm{~mm})$ & 2 \\
\hline & \multirow[t]{2}{*}{ 19-Shape } & \multirow[t]{2}{*}{ PWK } & Winged & 1 \\
\hline & & & Keeled & 2 \\
\hline \multirow[t]{8}{*}{ Seed } & \multirow[t]{2}{*}{ 20- Length } & \multirow[t]{2}{*}{ SL } & $(>2 \mathrm{~mm})$ & 1 \\
\hline & & & $(<2 \mathrm{~mm})$ & 2 \\
\hline & \multirow[t]{2}{*}{ 21- Width } & \multirow[t]{2}{*}{ SW } & $(>1 \mathrm{~mm})$ & 1 \\
\hline & & & $(<1 \mathrm{~mm})$ & 2 \\
\hline & \multirow[t]{4}{*}{ 22- Color } & \multirow[t]{4}{*}{$\mathrm{SC}$} & Black & 1 \\
\hline & & & Brown & 2 \\
\hline & & & Orange & 1 \\
\hline & & & Green & 2 \\
\hline \multirow[t]{2}{*}{ Seed/Pod } & \multirow[t]{2}{*}{ 23-Seed/Pod } & \multirow[t]{2}{*}{$\mathrm{S} / \mathrm{P}$} & $(>16 \mathrm{~mm})$ & 1 \\
\hline & & & $(<16 \mathrm{~mm})$ & 2 \\
\hline \multirow[t]{3}{*}{ Ovules } & \multirow[t]{3}{*}{ 24- Number } & \multirow[t]{3}{*}{$\mathrm{NOV}$} & $0-9$ & 1 \\
\hline & & & $10-19$ & 2 \\
\hline & & & $20-40$ & 3 \\
\hline
\end{tabular}

\section{RESULTS}

\section{Variations of characters among species}

Results of the basic descriptive statistics for quantitative continuous characters in all species are given in Table (4). None of the characters had a correlation coefficient above the threshold (0.90), and all characters show normal distribution where no transformations are performed (Tab. 5). Thus, all studied quantitative continuous (17) and quantitative discrete (7) characters are included in the analyses. The highest correlation coefficients -0.84 and 0.76 occurred between the characters style shape; STS vs. shape of lower leaflet; SLL and style length; STL vs. pod length; PL, respectively. The ANOVA test show that seven [Seed/pod length (4), seed color (7), shape of upper leaflet (8), shape of lower leaflet (11), calyx tube length (18), corolla color (21 and style shape (22)] out of the 24 examined characters are insignificantly different between species in all measured variables (Tab. 5). 
Gaafar et al.

Table (4): Basic descriptive statistics of quantitative parameters resulting from the morphometric analyses of the Lotus species ( $\mathrm{SD}=$ Standard deviation, $\mathrm{CV}=$ Coefficient of variation, 25\%-75\%=percentile boundaries. For species and character abbreviations see Tables (1) and (3), respectively).

\begin{tabular}{|c|c|c|c|c|c|c|c|c|c|c|c|c|c|c|c|c|c|c|c|c|}
\hline Species & & \begin{tabular}{|l}
$L$. \\
are
\end{tabular} & $\begin{array}{c}L . \\
e d u\end{array}$ & \begin{tabular}{|c}
$L$. \\
orn
\end{tabular} & $\begin{array}{c}\text { L. } \\
\text { halo }\end{array}$ & $\begin{array}{c}L . \\
\text { pere }\end{array}$ & $\begin{array}{c}. \\
\text { poly }\end{array}$ & $\begin{array}{c}\text { L. } \\
\text { cret }\end{array}$ & $\begin{array}{l}L . \\
c y t\end{array}$ & $\begin{array}{l}\text { L. } \\
\text { gla }\end{array}$ & $\begin{array}{c}L . \\
\text { ang }\end{array}$ & $\begin{array}{c}L . \\
\text { pal }\end{array}$ & $\begin{array}{c}L . \\
\text { glin }\end{array}$ & $\begin{array}{c}L . \\
\text { s. }\end{array}$ & $\begin{array}{c}\text { L. } \\
\text { arab }\end{array}$ & $\begin{array}{c}L . \\
\text { heb }\end{array}$ & $\begin{array}{c}L . \\
g e b\end{array}$ & $\begin{array}{c}\text { L. } \\
\text { lan }\end{array}$ & $\begin{array}{c}L . \\
\text { tetra }\end{array}$ & $\begin{array}{c}L . \\
\text { conj } \\
\end{array}$ \\
\hline \multicolumn{21}{|c|}{ Ouantitative continuous characters } \\
\hline \multirow{5}{*}{ PL } & & 3.6 & 5.1 & 35.0 & 22.5 & 35.1 & 12.5 & 2.5 & 5.0 & 25.0 & 7.5 & 19.1 & 6.1 & 1.0 & 27.5 & 22.5 & 25.0 & 18.5 & 5.0 & 45.0 \\
\hline & \begin{tabular}{|l|} 
SD \\
\end{tabular} & & & 6.0 & .7 & 5.5 & 1.6 & 8 & .4 & 6.9 & 7 & 2.8 & 0 & & 5.2 & 4.7 & 3.1 & 5.9 & 4.1 & 14.5 \\
\hline & & $7.3-$ & .6 & & & & 11.4- & & & & & & & & & & & & 9.4- & 1.7 \\
\hline & & 26.4 & 5.7 & 33.5 & 26.8 & 4.7 & 14.0 & 4.2 & 38.6 & 5.8 & 8.5 & 21.8 & 17.8 & 4.0 & 32.5 & 26.8 & 7.0 & 9.1 & 1.6 & \\
\hline & & 22.4 & & 5.1 & 1.0 & .9 & 12.6 & 4 & 9.8 & 4.6 & & 14.5 & & & & 1.0 & & 7 & 5.2 & 6.3 \\
\hline \multirow{5}{*}{$\mathrm{S} / \mathrm{P}$} & & .5 & 3.2 & 14.1 & 19.1 & 4.0 & 16.0 & 16.0 & 14.5 & 16.1 & 4.1 & 17.5 & 15.5 & .5 & 17.5 & 2.5 & 13.5 & 3.5 & 6.0 & 14.0 \\
\hline & SD & 3 & 1.0 & 2.3 & 5.0 & 5 & 0.8 & 0.8 & 1.6 & 0.8 & .7 & 0.4 & 0.4 & .0 & 1.6 & 6 & .0 & 2 & 3 & 0.7 \\
\hline & $25 \%-$ & $1.3-$ & 12.7 & $2.6-$ & $16.0-$ & $1.9-$ & 15.2- & 15.1- & 13.5- & $15.5-$ & $3.6-$ & 17.1- & 15.2 & & 16.3- & $0.8-$ & 12.4- & $2.2-$ & $4.8-$ & 13.4 \\
\hline & 75 & 23.9 & 8.8 & 15.4 & 21.0 & 5.5 & 16.7 & 5.6 & 15.6 & 16.9 & 4.6 & 17.8 & 15.9 & .2 & 18.5 & 23.6 & 14.4 & 4.3 & 6.7 & 14.6 \\
\hline & & 14.8 & & 16.3 & 26.5 & 7.7 & 4.9 & 9 & 11.3 & 4.7 & 7 & 2.3 & .7 & 13.0 & 9.4 & 3.7 & 7.7 & 8.7 & 4.5 & 5.0 \\
\hline \multirow{4}{*}{ SL } & & & & &. & & 1.2 & & 3 & & & & & & 1.5 & & & & & .5 \\
\hline & SD & 4 & 0.4 & 0.3 & 0.1 & 3 & 0.4 & 0.3 & 0.2 & 0.2 & & 0.2 & & .1 & 0.4 & & & & 6 & .4 \\
\hline & & \begin{tabular}{|l|}
$1.0-$ \\
1.3
\end{tabular} & $\begin{array}{l}2.5- \\
3.1\end{array}$ & $\begin{array}{l}1.8- \\
2.3\end{array}$ & $\begin{array}{l}0.9- \\
1.1\end{array}$ & $\begin{array}{l}3- \\
6\end{array}$ & $\begin{array}{l}1.2- \\
1.9\end{array}$ & $\begin{array}{l}1.4- \\
1.7\end{array}$ & $\begin{array}{l}1.1- \\
1.4\end{array}$ & $\begin{array}{l}1.1- \\
1.3\end{array}$ & $\begin{array}{l}8- \\
0\end{array}$ & $\begin{array}{l}1.5- \\
2.0\end{array}$ & $\begin{array}{l}1.1- \\
1.4\end{array}$ & \begin{tabular}{|l|}
$0.7-$ \\
0.9
\end{tabular} & $\begin{array}{l}1.1- \\
1.9\end{array}$ & $\begin{array}{l}1.0- \\
1.4\end{array}$ & $\begin{array}{l}.5- \\
.0\end{array}$ & $\begin{array}{l}1.5- \\
2.0\end{array}$ & $\begin{array}{l}.5- \\
.6\end{array}$ & $82-$ \\
\hline & \begin{tabular}{|l|}
$\mathrm{CV}$ \\
\end{tabular} & 33.7 & 4.7 & 16.8 & 14.9 & 22.0 & 25.7 & 19.1 & 15.7 & 13.2 & 1 & 12.4 & 15.2 & 15.6 & 25.9 & 20.0 & 12.4 & 12.4 & 21.0 & 14.7 \\
\hline \multirow{4}{*}{ SW } & & & 2.2 & 2.0 & & 9 & 1.0 & & 0 & 1.3 & & 1.0 & & 8 & 1.3 & 1.3 & 1.1 & & .5 & .8 \\
\hline & $\mathrm{S}$ & 5 & 0.3 & 0 & 0. & & 0.4 & & & & & & & & 0.2 & & & & & 2 \\
\hline & & & \begin{tabular}{|l}
$2.0-$ \\
2.4
\end{tabular} & & $\begin{array}{l}0.6- \\
1.0\end{array}$ & & & \begin{tabular}{|l|}
$1.1-$ \\
1.5
\end{tabular} & & $\begin{array}{l}1.1- \\
1.4\end{array}$ & & $\begin{array}{l}0.8- \\
1.2\end{array}$ & $\begin{array}{l}0.7- \\
0.9\end{array}$ & \begin{tabular}{|l|}
$0.6-$ \\
0.9
\end{tabular} & \begin{tabular}{|l|}
$1.1-$ \\
1.4
\end{tabular} & $\begin{array}{l}1.1- \\
1.4\end{array}$ & $\begin{array}{l}1.0- \\
1.2\end{array}$ & $\begin{array}{l}1.1- \\
1.4\end{array}$ & \begin{tabular}{|l|}
$2.3-$ \\
2.8
\end{tabular} & $\begin{array}{l}1.5- \\
2.0\end{array}$ \\
\hline & CV & 18.6 & $14 . .8$ & 23.0 & 28.3 & 10.5 & 41.9 & 16.1 & 15.1 & \begin{tabular}{|l}
14.7 \\
\end{tabular} & 1.1 & \begin{tabular}{|l|}
18.9 \\
\end{tabular} & 22.0 & 25.3 & 13.7 & 14.6 & 86 & 14.2 & 12.5 & 12.4 \\
\hline \multirow{5}{*}{ ULL } & lean & .4 & 1.5 & 12.5 & 5.5 & 13.5 & 5.3 & 10.0 & 10.0 & 11.0 & & 8.5 & 6.5 & 6.5 & 16.0 & 10.0 & 8.5 & 15.0 & 19.0 & 12.5 \\
\hline & & & & 3.4 & 1. & & 1. & & & 2.7 & & 1. & & & & & & & 7.3 & 1.5 \\
\hline & $25 \%-$ & 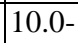 & $37-$ & () & 4.4- & & $3.8-$ & & & & & 7.7 & 3. & & 12.0- & 5.7- & & 11 & 12. & 118 \\
\hline & $75 \%$ & 12.0 & 5.3 & 4.9 & 6.8 & & 6.4 & 12.3 & & 13.1 & & 9.3 & & 7. & 19 & 14.7 & 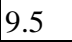 & & 24. & \\
\hline & \begin{tabular}{|l|}
$\mathrm{CV}$ \\
\end{tabular} & 16.1 & 2 & 26.9 & 28.9 & 16.9 & 25.6 & 32.0 & 30.7 & 24.5 & 26.7 & 11.7 & 42.2 & 14.6 & 26.9 & 46.6 & 26.0 & 24.6 & 38.5 & 12.4 \\
\hline \multirow{5}{*}{ ULJ } & Mean & & 7.0 & & 3.0 & & 7.5 & & & 4.1 & & 4.0 & 3.0 & & 7.5 & 7.5 & 4.0 & 6.5 & .0 & 6.0 \\
\hline & SD & 0.6 & 0.9 & & 0.8 & & 1.6 & & 2. & 1.2 & & 0.7 & 1. & 0.7 & 3. & 2.9 & 0.7 & 0.9 & 0.8 & 2.6 \\
\hline & $25 \%-$ & 6.0- & 6.3- & 7.7- & 2.3- & $6.2-$ & 6.5- & $3.6-$ & 3.3- & 3.1- & $3.1-$ & 3.4- & $1.2-$ & 3.6- & $4.7-$ & $\begin{array}{l}4.7- \\
0.5\end{array}$ & $3.6-$ & 6.1- & 6.3- & $4.2-$ \\
\hline & & 7.1 & 7.8 & 1.2 & 38 & & 8.2 & 8 & 7.1 & 5.2 & & 4.6 & 3.8 & 4.6 & 11.4 & 9.5 & & 7.1 & & \\
\hline & $\overline{\mathrm{CV}}$ & 9.6 & 13.1 & 36.4 & 25.5 & 21.4 & 21.9 & 40.1 & 40.6 & 30.1 & 29.4 & 17.2 & 46.7 & 17. & 52.6 & 38.1 & 17.6 & 13.4 & 10.9 & 43. \\
\hline \multirow{5}{*}{ LLL } & Mean & 3.3 & 7.0 & 6.1 & 5.0 & 6.5 & 3.5 & & 6.0 & 6.5 & 5.0 & 3.5 & & 3. & 10.0 & \begin{tabular}{|l|}
7.0 \\
\end{tabular} & 5.0 & 4.5 & 11.0 & 7.0 \\
\hline & SD & 0.6 & 0.5 & 1.7 & 1.9 & & 1.1 & & & 2.5 & & 0.9 & & & 6.0 & & & & & 2.2 \\
\hline & $25 \%-$ & $3.0-$ & $6.8-$ & $5.5-$ & $4.5-$ & $5.0-$ & $2.3-$ & $3.3-$ & $3.5-$ & $4.3-$ & $3.5-$ & 2.9- & $3.2-$ & 2.9 & $3.7-$ & $5.6-$ & $3.0-$ & $3.3-$ & $5.1-$ & 4.9 \\
\hline & & & & & & & & & & & & & & & & & & & & 3.4 \\
\hline & CV & & 7.0 & 27.0 & 38.2 & 31.0 & 30.8 & 33.6 & 46.4 & 38.0 & 39.6 & 24.8 & 18.0 & 28.3 & 60.3 & 25.8 & 40.0 & 33.6 & 43.3 & 31.0 \\
\hline \multirow{5}{*}{ LLW } & Mean & & & 5.5 & 2.5 & 5.0 & 2.0 & 4.1 & 3.8 & 2.0 & 2.0 & 2.1 & & 2. & 5.5 & 4. & 25 & 3.5 & 5.5 & .0 \\
\hline & \begin{tabular}{|l|}
$S D$ \\
\end{tabular} & 0.7 & 0.7 & 1.5 & 1.0 & 1.8 & 0.6 & 0.8 & 1.5 & 0.9 & 0.7 & 0.7 & 0.4 & 0.6 & 2.4 & 1.2 & .4 & 0.9 & 1.6 & 2.0 \\
\hline & $25 \%-$ & 1.9- & $4.8-$ & $4.9-$ & $1.8-$ & $3.9-$ & 1.6- & $3.1-$ & 2.9- & 1.1- & 1.3- & $1.5-$ & 2.1- & $1.5-$ & 4.1- & $3.1-$ & $2.1-$ & 2.9- & 4.1- & 3.4 \\
\hline & & & & & & & & & & 2.0 & & 2.6 & 2.8 & & 1.5 & 5.0 & 2.9 & 4.2 & 6.5 & 1.1 \\
\hline & & 30.1 & 14.3 & 26.7 & 39.1 & 35.5 & 32.1 & 20.7 & 40.2 & 42.4 & 36.4 & 33.9 & 14.6 & 32.1 & 43.4 & 30.1 & 15.7 & 24.8 & 29.2 & 39.8 \\
\hline & & 8.1 & 7. & 5.0 & 2.0 & 5.5 & 2.5 & \begin{tabular}{|l|}
0.8 \\
\end{tabular} & \begin{tabular}{|l|}
2.8 \\
\end{tabular} & 3.5 & 4.5 & \begin{tabular}{|l|}
6.5 \\
\end{tabular} & 3.5 & & 5.0 & 2.8 & 10.0 & \begin{tabular}{|l|}
3.0 \\
\end{tabular} & \begin{tabular}{|l|}
7.5 \\
\end{tabular} & 8.5 \\
\hline & SD & 0.8 & 2.0 & 0.7 & 0.6 & 1.6 & 0.4 & 0.2 & 1.7 & 1.1 & 1.0 & 2.4 & 1.0 & 1.0 & 2.0 & 1.2 & 2.3 & 0.8 & 1.5 & 1.2 \\
\hline
\end{tabular}


A multivariate morphometric analysis

\begin{tabular}{|c|c|c|c|c|c|c|c|c|c|c|c|c|c|c|c|c|c|c|c|c|}
\hline & $\begin{array}{l}25 \%- \\
75 \%\end{array}$ & $\begin{array}{l}7.5- \\
8.5 \\
\end{array}$ & $\begin{array}{r}5.3- \\
8.5 \\
\end{array}$ & $\begin{array}{r}4.4- \\
5.6 \\
\end{array}$ & $\begin{array}{l}1.5- \\
2.5\end{array}$ & $\begin{array}{c}4.4- \\
6.1 \\
\end{array}$ & $\begin{array}{l}2.0- \\
2.9\end{array}$ & $\begin{array}{c}0.6- \\
0,8\end{array}$ & $\begin{array}{c}0.8- \\
4.2 \\
\end{array}$ & $\begin{array}{c}2.8- \\
4.8 \\
\end{array}$ & $\begin{array}{c}3.8- \\
5.4 \\
\end{array}$ & $\begin{array}{r}4.4- \\
8.5 \\
\end{array}$ & $\begin{array}{c}2.9- \\
4.1 \\
\end{array}$ & $\begin{array}{c}2.9- \\
4.1 \\
\end{array}$ & $\begin{array}{l}3.4- \\
7.1\end{array}$ & $\begin{array}{l}1.5- \\
3.9\end{array}$ & $\begin{array}{l}8.0- \\
11.4\end{array}$ & $\begin{array}{l}2.0- \\
4.0 \\
\end{array}$ & $\begin{array}{c}6.4- \\
8.7 \\
\end{array}$ & $\begin{array}{l}7.7- \\
9.3\end{array}$ \\
\hline & $\mathrm{CV}$ & 9.6 & 28.1 & 13.6 & 0.2 & 28.4 & 16.1 & 22.0 & 60.5 & 32.4 & 22.8 & 37.5 & 28.3 & 28.3 & 39.8 & 44.5 & 23.3 & 27.2 & 19.9 & 14.7 \\
\hline \multirow{4}{*}{ BL } & Tean & 9.0 & 7.0 & 9.5 & 5.5 & 6.5 & 5.5 & 5.5 & 4.5 & 6.5 & 7.0 & .5 & 4.0 & 3.0 & 9.0 & 9.0 & 10.0 & 5.0 & 0.0 & 11.0 \\
\hline & $\mathrm{D}$ & 1.0 & 1.3 & .0 & 1.6 & .1 & 0.4 & .4 & 1.0 & 1.1 & .8 & .4 & .7 & .6 & .7 & .7 & 0.7 & 0.7 & 2.6 & 1.5 \\
\hline & $\begin{array}{l}5 \%- \\
5 \%\end{array}$ & $\begin{array}{l}8.2- \\
9.5\end{array}$ & $\begin{array}{c}6.1- \\
7.2\end{array}$ & & $\begin{array}{c}4.1- \\
5.6\end{array}$ & $\begin{array}{l}6.0- \\
7.3\end{array}$ & $\begin{array}{c}5.1- \\
6.0\end{array}$ & $\begin{array}{c}5.2- \\
5.7\end{array}$ & $\begin{array}{c}3.7- \\
5.2\end{array}$ & $\begin{array}{c}5.5- \\
7.2\end{array}$ & $\begin{array}{l}6.3- \\
7.8\end{array}$ & $\begin{array}{c}9.2- \\
9.8\end{array}$ & $\begin{array}{c}3.6- \\
4.7\end{array}$ & $\begin{array}{c}2.5- \\
3.6\end{array}$ & $\begin{array}{c}8.4- \\
9.3\end{array}$ & $\begin{array}{l}8.3- \\
9.5\end{array}$ & $\begin{array}{l}9.4- \\
10.6\end{array}$ & $\begin{array}{l}4.3- \\
5.6\end{array}$ & $\begin{array}{c}7.0- \\
12.6\end{array}$ & $\begin{array}{l}9.8- \\
12.4\end{array}$ \\
\hline & $\mathrm{V}$ & 11.1 & 18.0 & 10.8 & 29.2 & 16.4 & 7.3 & 6.5 & 22.0 & 16.5 & 11.1 & 4.0 & 16.8 & 21.0 & 7.4 & 0.8 & 6.9 & 14.7 & 25.7 & 13.4 \\
\hline \multirow{4}{*}{$\mathrm{NF}$} & Mean & 4.3 & 1.5 & 3.6 & 2.5 & 2.0 & 4.0 & 4.5 & 5.0 & 2.0 & 2.0 & 3.5 & 3.0 & 3.0 & 3.0 & 3.5 & 3.5 & 3.0 & 1.5 & 1.5 \\
\hline & SD & 0.5 & 0.5 & 1.0 & 0.5 & 0.8 & 1.2 & 1.1 & 2.0 & 0.7 & 0.8 & 1.6 & 0.9 & 0.8 & 0.9 & 0.5 & 1.1 & 0.9 & .5 & 0.5 \\
\hline & $5 \%-$ & $\begin{array}{l}0- \\
0\end{array}$ & $\begin{array}{l}1.0- \\
2.0\end{array}$ & $\begin{array}{c}3.0- \\
4.0\end{array}$ & $\begin{array}{c}2.0- \\
3.0\end{array}$ & $\begin{array}{l}1.0- \\
3.0\end{array}$ & $\begin{array}{c}3.0- \\
5.0\end{array}$ & $\begin{array}{c}4.0- \\
5.0\end{array}$ & $\begin{array}{l}3.0- \\
7.0\end{array}$ & $\begin{array}{c}2.0- \\
2.0\end{array}$ & $\begin{array}{l}1.5- \\
3.0\end{array}$ & $\begin{array}{c}2.0- \\
5.0\end{array}$ & $\begin{array}{c}2.0- \\
4.0\end{array}$ & $\begin{array}{c}2.0- \\
4.0\end{array}$ & $\begin{array}{c}2.0- \\
4.0\end{array}$ & $\begin{array}{c}3.0- \\
4.0\end{array}$ & & $\begin{array}{c}2.0- \\
4.0\end{array}$ & $\begin{array}{l}5- \\
.0\end{array}$ & \\
\hline & & 1.2 & 35.1 & 26.8 & 1.1 & 40.8 & 28.9 & 24.0 & 40.0 & 33.3 & 40.8 & 45.2 & 31.4 & 27.2 & 31.4 & 15.1 & 30.9 & 31.4 & 5.1 & 0.1 \\
\hline \multirow{5}{*}{ CL } & Iean & 8.0 & 8.5 & 6.5 & 5.1 & 6.5 & 5.5 & 8.0 & 7.5 & 5.5 & 5.5 & 7.5 & 4.0 & 4.0 & 7.5 & 6.5 & 8.5 & 7.5 & 12.5 & 12.5 \\
\hline & \begin{tabular}{|l|} 
SD \\
\end{tabular} & 0.7 & 1.3 & 1.0 & 0.7 & 1.1 & 0.8 & 0.6 & 1.1 & 1.1 & 0.4 & 1.1 & 0.7 & 0.7 & 1.0 & 0.9 & 1.0 & 1.1 & 2.7 & 1.6 \\
\hline & $5 \%-$ & 7- & $7.9-$ & $5.7-$ & $4.3-$ & $5.7-$ & $5.0-$ & $7.5-$ & $6.4-$ & $4.7-$ & $5.2-$ & $6.4-$ & $3.6-$ & $.6-$ & $6.6-$ & $6.1-$ & $7.7-$ & $5.6-$ & $9.9-$ & 11.4 \\
\hline & $5 \%$ & 8.5 & 9.2 & 7.4 & 5.2 & 7.5 & 6.1 & 8.5 & 8.4 & 6.4 & 5.9 & 8.5 & 4.5 & 4.5 & 8.2 & 7.1 & 9.3 & 8.2 & 15.3 & 14. \\
\hline & & 6 & 5.8 & 4.8 & 4.8 & 17.0 & 5.1 & 8.0 & 15.2 & $\overline{9.3}$ & 7.2 & 14.6 & 17.3 & 6.8 & 13.7 & 3.4 & 11.7 & 4.5 & 21.3 & 12.5 \\
\hline \multirow{5}{*}{ CTU } & eal & & 3. & 3.' & 2.5 & .5 & 2. & 3.5 & 3. & 3.0 & 1.5 & 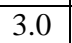 & 2.5 & & 3.0 & 3.0 & 4.3 & 3.0 & 4.0 & 8.0 \\
\hline & $\mathrm{D}$ & 0.7 & 0.5 & 0.9 & 0.4 & 0.4 & 0.4 & 0.4 & 0.4 & 0.4 & 0.4 & 0.4 & 0.3 & 0.5 & 0.7 & 0.9 & 0.6 & 0.8 & 0.7 & 1.7 \\
\hline & $25 \%-$ & $3.0-$ & $3.1-$ & $2.9-$ & $2.1-$ & $3.2-$ & $2.1-$ & $3.1-$ & $3.1-$ & 2.6- & 1.1- & 2.6- & 2.3- & 1.9- & $3.0-$ & $2.0-$ & 3.7- & $2.0-$ & 3.4- & $6.4-$ \\
\hline & $5 \%$ & 4.2 & 4.0 & 4.4 & 3.0 & $4 . .0$ & 2.9 & 3.8 & 3.9 & 3.2 & 1.9 & 3.2 & 2.8 & 2.7 & 3.0 & 4.0 & 4.9 & 4.0 & 4.5 & 9.8 \\
\hline & $\overline{\mathrm{CV}}$ & 18.4 & 3.4 & 23.0 & 16.5 & 11.6 & 15.7 & 11.9 & 11.1 & 12.5 & 25.9 & 12.7 & 12.5 & 4.4 & 22.2 & 31.4 & 14.0 & 27.2 & 17.0 & 21.5 \\
\hline \multirow{5}{*}{ CT } & 1 & .0 & & & & 30 & 3. & 45 & & 3 & 3.5 & & 25 & & 5.0 & & 4.3 & \begin{tabular}{|l|}
4.5 \\
\end{tabular} & 8.5 & 4.0 \\
\hline & & 0 & 0 & & & & & & & & .4 & & & & & & & .4 & & .7 \\
\hline & $25 \%-$ & $5.0-$ & $.0-$ & $2.5-$ & 1.0- & $2.0-$ & $2.0-$ & $4.0-$ & $3.0-$ & $2.7-$ & 3.1- & $3.9-$ & $2.2-$ & .6- & $4.2-$ & $2.0-$ & 3.8- & $4.2-$ & 6.9- & $.4-$ \\
\hline & & & & & 2.0 & 4.0 & 4.0 & 5.0 & & 3.5 & 3.8 & 5.0 & 2.8 & 2.0 & 5.7 & 4.0 & 4.9 & 4.9 & 9.8 & 4.5 \\
\hline & $\mathrm{V}$ & .0 & & 8.2 & 35.1 & 31.4 & 27.2 & 1.7 & 20.4 & 3.3 & 11.3 & 15.0 & 14.7 & 1.2 & 15.5 & 31.4 & 14.9 & 8.2 & 21.0 & 17.0 \\
\hline \multirow{5}{*}{ CRL } & Iean & & & & & 9.0 & 6.5 & 15.0 & 11.0 & & & & & & 8.5 & 9.5 & 12.9 & 14.0 & 16.0 & 13. \\
\hline & & & & & & & & 2.0 & & & 9 & & & & 0.9 & .0 & 2.5 & 3.1 & 2.5 & 1.2 \\
\hline & $25 \%-$ & $2.1-$ & .6- & 9.4- & $5.5-$ & $8.6-$ & $5.7-$ & 3.8 & 9 & $7.8-$ & $6.1-$ & 8.4- & $5.7-$ & $3.2-$ & $7.8-$ & $7.5-$ & $12.0-$ & $11.8-$ & 13.5 & 12.2 \\
\hline & & 3.0 & & & & & 7 & 5.6 & & & & & 6 & & 9.1 & & & 6.4 & & 14.3 \\
\hline & \begin{tabular}{|l|}
$\mathrm{CV}$ \\
\end{tabular} & 3.4 & 12.3 & 6.4 & 17.6 & 7.1 & 15.2 & 13.2 & 19.1 & 10.7 & \begin{tabular}{|l|}
13.4 \\
\end{tabular} & 7.6 & 9.0 & 18.7 & 10.9 & 21.0 & \begin{tabular}{|l}
19.7 \\
\end{tabular} & 21.9 & 15.7 & 8.7 \\
\hline \multirow{5}{*}{ STL } & Mean & & 5.5 & 3.3 & 2.3 & 2.8 & 2.8 & 6.6 & 4.5 & 3.5 & 2.8 & 5.0 & 1.3 & 1.8 & 3.5 & 4.8 & 6.5 & 5.5 & 3.5 & 6.0 \\
\hline & \begin{tabular}{|l|} 
SD \\
\end{tabular} & 0.4 & 0.4 & 0.2 & 0.3 & 0.2 & 0.2 & 0.4 & & 0.3 & 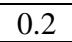 & 0.5 & 0.2 & & 0.4 & 0.2 & 0.4 & 0.4 & .4 & 0.8 \\
\hline & $25 \%$ & $65-$ & $5.0-$ & $3.0-$ & $2.1-$ & $2.5-$ & $2.5-$ & $6.2-$ & 4 & $3.3-$ & $2.6-$ & $4.8-$ & $1.1-$ & $1.5-$ & $3.1-$ & $4.5-$ & $6.1-$ & $5.2-$ & $3.1-$ & 5.5 \\
\hline & & & & & & & & & & & & & & & & & & 0.0 & & 6.4 \\
\hline & $\mathrm{CV}$ & & & & & 7.9 & & 6.3 & 10.0 & 9.4 & 7.3 & 9.6 & 13.7 & 12.4 & 10.9 & 4.8 & 5.7 & 6.9 & 1.9 & 12.8 \\
\hline \multirow{5}{*}{$\mathrm{NOV}$} & Mean & 39.3 & 16.0 & 25.9 & 18.0 & 29.5 & 19.0 & 16.0 & 20.0 & 24.0 & 16.0 & 16.5 & 16.9 & 8.0 & 21.0 & 29.5 & 34.0 & 34.0 & 18.4 & 16.0 \\
\hline & \begin{tabular}{|l|} 
SD \\
\end{tabular} & 4.4 & 0.8 & 0.9 & 0.8 & 1.3 & 0.8 & 0.8 & 0.9 & 1.8 & 0.8 & 1.1 & 0.9 & 0.8 & 0.9 & 1.1 & 0.7 & $\begin{array}{l}0.9 \\
\end{array}$ & 1.3 & 0.8 \\
\hline & $25 \%-$ & $37.0-$ & $15.0-$ & $25.0-$ & 17.0 & $28.0-$ & $18.0-$ & $15.0-$ & $19.0-$ & 23.0 & $15.0-$ & 16.0- & 16.0- & $7.0-$ & $20.0-$ & $29.0-$ & $34.0-$ & $33.0-$ & $17.0-$ & 15.0 \\
\hline & & 41.0 & 17.0 & 27.0 & 19.0 & 31.0 & 20.0 & 17.0 & 21.0 & 25.0 & 17.0 & 17.0 & 18.0 & 9.0 & 22.0 & 30.0 & 34.0 & 35.0 & 20.0 & 17.0 \\
\hline & & 11.2 & 5.1 & 4.3 & 4.5 & 4.3 & 4.3 & 5.1 & \begin{tabular}{|l|}
4.7 \\
\end{tabular} & 7.6 & 5.1 & 6.5 & 5.2 & 10.2 & 4.5 & 3.7 & 2.0 & \begin{tabular}{|l|}
2.8 \\
\end{tabular} & 6.9 & 5.1 \\
\hline
\end{tabular}


Gaafar et al.

Table (5): Results of ANOVA for the means of 24 morphological characters between the species groups resulted from cluster analysis of Lotus species (For full names of character abbreviations, see Table (3). NS $=$ Not significant, $*=P<0.05, * *=$ $P<0.01)$.

\begin{tabular}{|c|c|c|c|c|c|c|c|c|}
\hline & \multicolumn{6}{|c|}{ Species groups } & \multirow{3}{*}{$\begin{array}{c}\text { F- } \\
\text { value }\end{array}$} & \multirow{3}{*}{$\begin{array}{c}\text { Total } \\
P\end{array}$} \\
\hline & I & II & III & IV & $\mathrm{V}$ & VI & & \\
\hline $\begin{array}{l}\text { Number } \\
\text { of species }\end{array}$ & 4 & 2 & 3 & 3 & 3 & 4 & & \\
\hline \multicolumn{9}{|c|}{ Quantitative cardinal characters } \\
\hline $\mathrm{H}$ & NS & NS & NS & $0.001 * *$ & NS & NS & 13.41 & $0.001 * *$ \\
\hline PWK & NS & NS & NS & NS & $0.03 *$ & NS & 3.28 & $0.039 *$ \\
\hline $\mathrm{SC}$ & NS & NS & NS & $0.001 * *$ & NS & $0.001 * *$ & 1.07 & 0.42 \\
\hline SUL & $0.001 * *$ & NS & $0.001 * *$ & $0.001 * *$ & $0.001 * *$ & $0.001 * *$ & 1.35 & 0.30 \\
\hline SLL & $0.04 *$ & NS & $0.001 * *$ & $0.001 * *$ & $0.001 * *$ & NS & 1.20 & 0.36 \\
\hline CRC & $0.04 *$ & NS & NS & $0.001 * *$ & $0.001 * *$ & NS & 0.85 & 0.54 \\
\hline STS & $0.001 * *$ & NS & NS & $0.001 * *$ & NS & NS & 1.83 & 0.17 \\
\hline \multicolumn{9}{|c|}{ Quantitative continuous characters } \\
\hline PL & NS & NS & NS & NS & $0.001 * *$ & NS & 3.04 & $0.049 *$ \\
\hline $\mathrm{S} / \mathrm{P}$ & NS & NS & $0.001 * *$ & $0.001 * *$ & NS & NS & 0.40 & 0.084 \\
\hline SL & NS & NS & $0.001 * *$ & $0.001 * *$ & NS & NS & 19.64 & $0.001 * *$ \\
\hline SW & $0.001 * *$ & NS & $0.001 * *$ & NS & NS & NS & 8.56 & $0.001 * *$ \\
\hline ULL & NS & NS & $0.001 * *$ & NS & NS & NS & 5.11 & $0.008 * *$ \\
\hline ULW & $0.04 *$ & NS & $0.001 * *$ & $0.001 * *$ & $0.001 * *$ & NS & 10.98 & $0.001 * *$ \\
\hline LLL & NS & NS & NS & NS & $0.001 * *$ & NS & 4.10 & $0.019 *$ \\
\hline LLW & $0.04 *$ & NS & NS & NS & NS & NS & 14.98 & $0.001 * *$ \\
\hline $\mathrm{R}$ & NS & $0.02 *$ & NS & NS & NS & NS & 4.42 & $0.014 *$ \\
\hline $\mathrm{BL}$ & NS & NS & $0.001 * *$ & NS & NS & NS & 3.52 & $0.031 *$ \\
\hline $\mathrm{NF}$ & $0.05^{*}$ & NS & NS & NS & NS & NS & 9.00 & $0.001 * *$ \\
\hline CL & NS & NS & NS & NS & $0.001 * *$ & $0.001 * *$ & 10.78 & $0.003 * *$ \\
\hline CTU & NS & NS & $0.001 * *$ & NS & NS & NS & 2.72 & 0.07 \\
\hline $\mathrm{CT}$ & NS & NS & NS & NS & NS & $0.025^{*}$ & 3.54 & $0.031 *$ \\
\hline CRL & NS & NS & NS & NS & NS & NS & 8.60 & $0.001 * *$ \\
\hline STL & NS & NS & NS & $0.003 * *$ & NS & NS & 5.71 & $0.005 * *$ \\
\hline NOV & NS & NS & NS & $0.001 * *$ & $0.001 * *$ & NS & 15.06 & $0.001 * *$ \\
\hline
\end{tabular}

\section{Cluster analysis}

The UPGMA dendrogram (Diag. 1) is based on morphological similarity values (Gower's coefficient) with a cophenetic correlation of 0.704 , demonstrating good consistency in the presented morphological patterns. The tree can be divided into three acceptable levels yielding six species-specific groups, beyond which recognition of the larger number of groups will be less significant. At the first level of classification, groups (V) and (VI) were separated. Group (V) comprised of Lotus edulis L. (Sect. Krokeria), L. tetragonolobus L. and L. conjugatus L. (the latter two species included in Subgenus Tetragonolobus), and group (VI) included Lotus ornithopodioides L., L. peregrinus L., L. arabicus L. and L. hebranicus Hochst. ex Brand. At the second hierarchical level, groups (III) and (IV) were recognized. Group (III) comprised of the perennials Lotus polyphyllos E.D. Clarke, L. creticus L. and $L$. cytisoides L., group (IV) consisted of $L$. gebelia Vent., L. lanuginosus Vent. and $L$. arenarius Brot. (subgenus Pedrosia). At the third classification level, groups (I) and (II) were separated. 
Group (I) consisted of OUT's of Lotus halophilus Boiss. \& Spruner, L. angustissimus L., L. glinoides Delile and L. schimperi Steud. ex Boiss., group (II) included the perennials $L$. glaber Mill. and L. palustris Willd. The results of ANOVA test between the six separated groups (I-VI) showed that habit of the plant $(\mathrm{H} ; p=0.001)$ and pod shape $(\mathrm{PWK} ; p=0.039)$ were the quantitative discrete cardinal characters that were significantly different among groups (Tab. 6). Except for the length of calyx tube (CTU) and the ratio between seed/pod $(\mathrm{S} / \mathrm{P})$, all the remaining quantitative continuous characters were significantly different among groups.

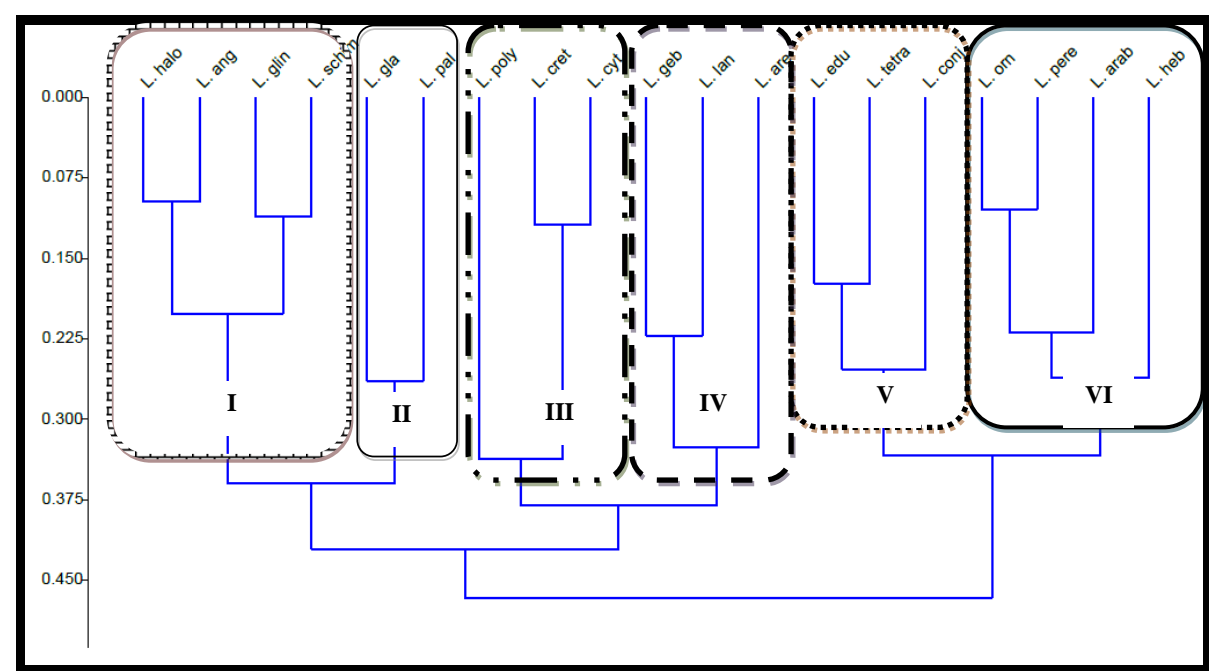

Diagram (1): Cluster analysis (UPGMA classification method and Gower's similarity coefficient) derived from the 24 characters of studied Lotus species (Species abbreviations are shown in Table (1), I-VI are the separated species groups).

Table (6): Pearson's correlations coefficients between characters (For full names of character numbers, see Table (3). $* *=p<0.01, *=p<0.05)$.

\begin{tabular}{|c|c|c|}
\hline 2 & -0.05 & \\
\hline 3 & -0.210 .42 & \\
\hline 4 & 0.220 .280 .24 & \\
\hline 5 & -0.350 .410 .630 .07 & \\
\hline 6 & 0.460 .280 .220 .270 .35 & \\
\hline 7 & $\begin{array}{lll}0.01 & 0.340 .030 .17 & 0.02\end{array}$ & 0.17 \\
\hline 8 & $0.20 \overline{-}_{0.240 .45^{0.09-0.40}}^{-}$ & 0.090 .00 \\
\hline 9 & $-0.190 .150 .09^{-} 0.420 .29$ & 0.190 .160 .01 \\
\hline
\end{tabular}


10

11

12

13

14

15

16

17

18

19

20

21

22

23

24

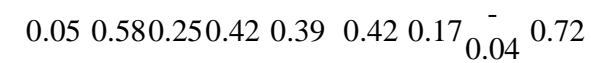
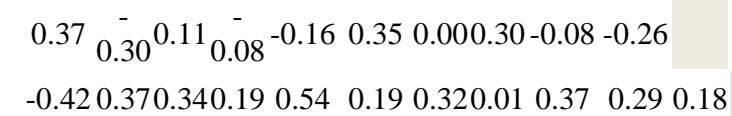

\section{Principal Coordinates Analysis (PCoA)}

Based on Gower's similarity coefficient of the 24 characters, a principal coordinates analysis (PCoA) is performed and visualized in Diagram (2). It supports the separation patterns of the six species groups (I-VI) along the first two axes that are responsible for $40.7 \%$ of the total variation (26.25\% for axis 1 , and $14.45 \%$ for axis 2 ). A clear separation between groups (I) and (II) positioned along the negative end of axis 1, and groups (V) and (VI) along its positive ends was indicated. An overlap occurred between groups (III) and (IV) positioned along the negative end of axis 2. Other projections confirmed the same general pattern, although less clearly because they are supported by axes that account for less inertia than the first two. Along axis 1 (results not shown), characters with the highest scores (more than 0.6) were pod length (PL), seed length (SL), upper leaflet width (ULW), lower leaflet width 
(LLW), number of flowers (NF), calyx length (CL) and calyx tube length (CTU). Along axis 2, the habit of plants $(\mathrm{H})$ and style shape (STS) had the highest scores. Thirteen out of the 17 quantitative continuous characters showed significant variations along the first three PCA axes (Tab. 7). Variations between PL, S/P, R and CT were insignificantly different along the three axes.

\section{Principal Components Analysis (PCA)}

The ordination diagram from the principal components analysis (Diag. 3) based on the 17 quantitative continuous characters showed a pattern similar to the results of the cluster analysis. The scores of the first three components explained $61.1 \%$ of the total variation accounted for $34.2 \%, 14.5 \%$, and $12.4 \%$ of the total variance for axes 1, 2 and 3, respectively. Pod length (PL), seed width (SW), upper leaflet width (ULW), lower leaflet width (LLW), calyx length (CL), and calyx tube length (CTU) showed highest loadings in relation to PCA axis 1 (Tab. 7). Along PCA axis 2, length of lower leaflet (LLL), length of corolla (CRL) and length of style (STL) had the highest loadings (the latter character contributed weakly to PCA axis 1).

The seed length (SL), number of flowers (NF) and number of ovules (NOV) contributed essentially to the construction of PCA axis 3. The six species groups were distributed in the ordination plane, with some overlap, along the first two important PCA axes. Inspection to the PCA diagram, the number of flowers (NF) was correlated to group (I) that occupied the positive end of PCA axis 1, while groups (V) and (VI) occupied the negative end that were correlated to (LLL), (LLW), (ULW) and (CL). Whereas group (II), which occupied the center of the ordination plane, was not affected by any character, groups (III) and (IV) that positioned on the positive end of PCA axis 2 were correlated with (STL).

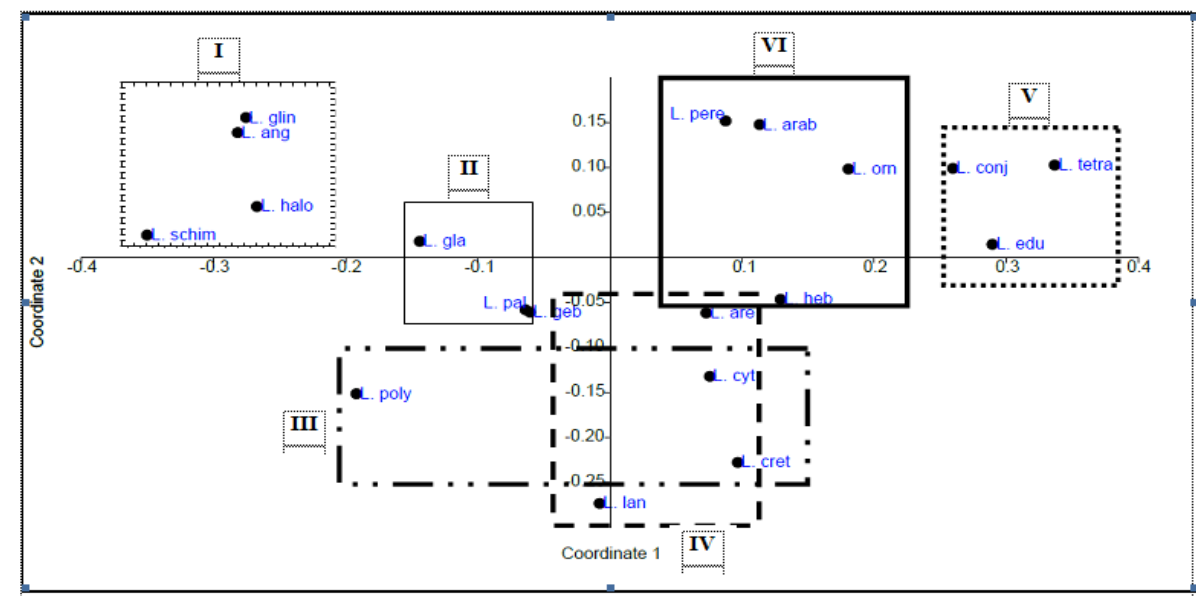

Diagram (3): Scatter plot of principal components analysis (PCA) performed on 17 quantitative characters along the first two PCA axes, with projection of the variables on the factor plane (For species and character abbreviations see Tables (1) and (3), respectively. Gr I-VI refers to the species groups). 
Gaafar et al.

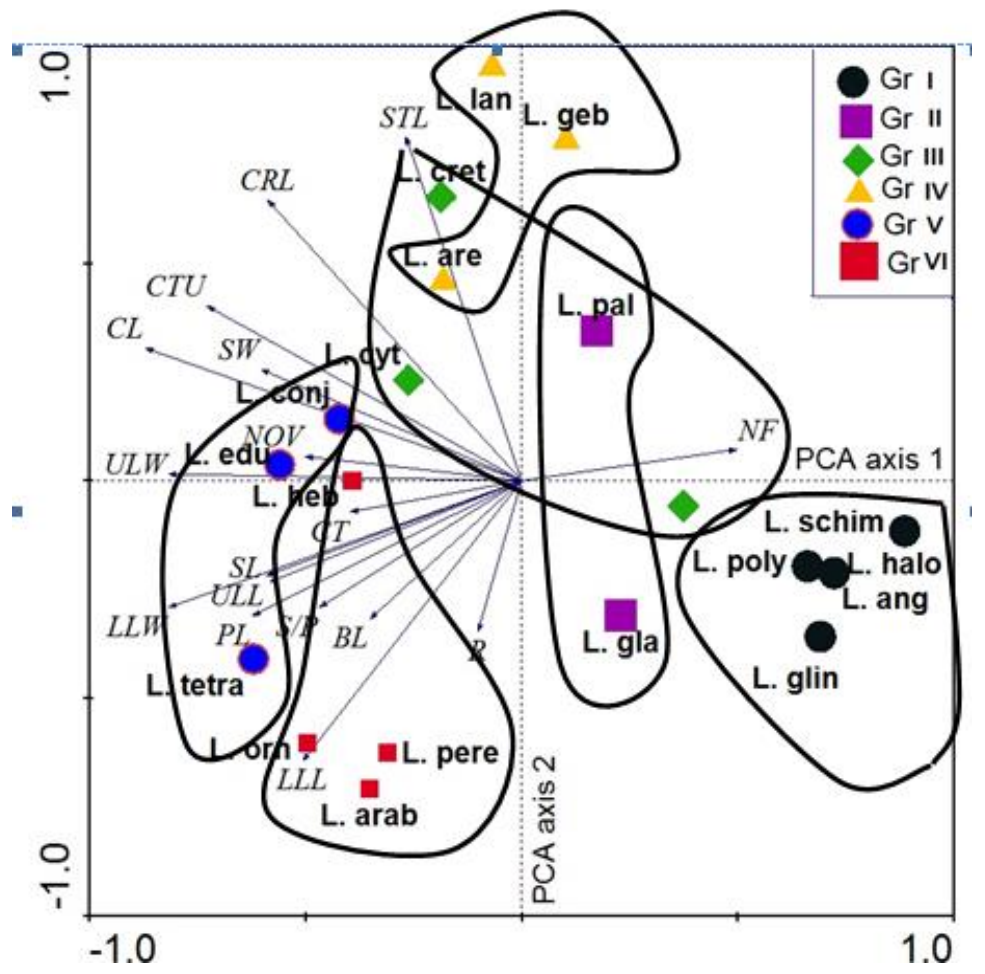

Diagram (2): Principal coordinates analysis (PCoA) scatterplot performed on 24 quantitative and qualitative characters along axes 1 and 2, I-VI are the species groups, for species abbreviations see Table (1).

Table (7): Results of the principal components analysis (PCA) for the species of the Lotus as OTUs-total variance and 17 morphological quantitative continuous characters showing the factor loadings on the first three principal components, and results of one-way ANOVA F- and P-values for characters with normal distribution (The numbers in bold are characters with high factor loading $>0.6$. For character abbreviations, see Table $(3), *=P<0.05, * *=P<0.01)$.

\begin{tabular}{lccccc}
\hline \multirow{2}{*}{ Characters } & \multicolumn{3}{c}{ PCA-factor loadings } & \multirow{2}{*}{ ANOVA-F value } & \multirow{2}{*}{$P$ value } \\
\cline { 2 - 4 } & PC1 & PC2 & PC3 & & \\
\hline PL & $\mathbf{- 0 . 6 2}$ & -0.31 & 0.04 & 2.92 & 0.056 \\
S/P & -0.47 & -0.29 & -0.38 & 2.52 & 0.083 \\
SL & -0.58 & -0.23 & $\mathbf{0 . 6 1}$ & 8.35 & $0.001^{* *}$ \\
SW & $\mathbf{- 0 . 6 3}$ & 0.26 & -0.02 & 4.93 & $0.009^{* *}$ \\
ULL & -0.59 & -0.22 & -0.33 & 3.56 & $0.030^{*}$ \\
ULW & $\mathbf{- 0 . 8 1}$ & 0.01 & -0.21 & 14.64 & $0.001^{* *}$ \\
LLL & -0.50 & $\mathbf{- 0 . 6 4}$ & 0.17 & 7.25 & $0.002^{* *}$ \\
LLW & $\mathbf{- 0 . 8 2}$ & -0.29 & 0.08 & 15.88 & $0.001^{* *}$ \\
R & -0.10 & -0.35 & 0.47 & 0.78 & 0.581 \\
BL & -0.35 & -0.32 & 0.17 & 3.80 & $0.024^{*}$
\end{tabular}


A multivariate morphometric analysis

\begin{tabular}{lccccc} 
NF & 0.50 & 0.07 & $\mathbf{- 0 . 7 6}$ & 6.38 & $0.003^{* *}$ \\
CL & $\mathbf{- 0 . 8 7}$ & 0.30 & 0.0007 & 6.55 & $0.003^{* *}$ \\
CTU & $\mathbf{- 0 . 7 3}$ & 0.40 & 0.02 & 4.93 & $0.009^{* *}$ \\
CT & -0.40 & -0.07 & 0.30 & 1.11 & 0.403 \\
CRL & -0.59 & $\mathbf{0 . 6 4}$ & 0.16 & 6.09 & $0.004^{* *}$ \\
STL & -0.27 & $\mathbf{0 . 7 9}$ & 0.33 & 3.67 & $0.03^{*}$ \\
NOV & -0.50 & 0.06 & $\mathbf{- 0 . 6 7}$ & 6.40 & $0.003^{* *}$ \\
\hline
\end{tabular}

\section{DISCUSSION}

The genus Lotus possesses a difficult generic delimitation, the classification of this genus is always of controversy among taxonomists; Whereas Gillett (1958) and Ball and ChrtkováŽertová (1968) proposed subgenera, sections and subsections. On the other hand, Heyn (1970) and Heyn and Herrnstadt (1968) suggested species groups to place the taxa and describe the relationships among species. This study of Lotus species in Egypt was based on the results of numerical analyses of morphological characters (vegetative and reproductive), the current results showed significant characters that may help in the diagnosis of the studied taxa, which were significantly different concerning the analyzed morphological characters.

In the present study, the applications of multivariate morphometric techniques resulted in the delimitation of 19 well-separated species of Lotus, and are clearly distinguished from each other. As a result of the cluster, PCoA, and PCA analyses six clear clusters are obtained and they correspond quite well with the species of Lotus accepted in Flora of Egypt (El Hadidy, 2003; Boulos, 2009; El-Gazzar et al., 2013). UPGMA gives insight into the degree of similarity among the OUT's and whether they form groups/clusters. PCoA and PCA reflect which characters are important on the axes, and indicate the significant characters based on the highest factor loadings. For that reason, it becomes clear which characters are diagnostic and support the separation between groups, and can be useful to distinguish taxa. This study revealed the importance of pod length, seed dimensions, measurements of upper and lower leaflets, calyx, length of corolla, length of style, numbers of flowers and ovules as characteristics that determinate the studied 19 species of Lotus. Generally, our results confirm congruence between the UPGMA clustering, PCoA and PCA analyses, in suggesting six groups:

\section{Group (I): Lotus halophilus, L. angustissimus, L. glinoides and L. schimperi}

This group can be differentiated from the others by the width of the lower leaflets (LLW) and the variations in the number of flowers (NF) which showed significant differences within members of this group ( $P=0.04$ and 0.05 , respectively), and among other groups $(P=0.001$; Tab. 6). Despite not being included in the analysis, the morphological difference in the pod shape (PS) between species of this group was diagnostic: curved in L. glinoides but straight in the others. This group also occupied the extreme ends along the first axes of PCoA and PCA. Other significant quantitative continuous characters were seed width (SW), and upper leaflets width (ULW). Along PCA axis 1, it occupied the positive end that was affected significantly by the number of flowers (NF). 


\section{Group (II): Lotus glaber and L. palustris}

Both species are annuals (Sect. Lotus) and can be distinguished by the length of rachis (R) that showed significant difference $(P=0.02)$ between them $(3.5 \mathrm{~mm}$ for the former and $6.5 \mathrm{~mm}$ for the latter), and among other groups $(P=0.014)$. In PCoA ordination diagram, this group occupied a central position along axis 1 , and was not affected by any character in PCA diagram. The number of lateral veins, not included in the analysis, can be used to distinguish both species from each other: 3 pairs in L. glaber, and 2 pairs in L. palustris. According to Zareh et al. (2017), Lotus glaber can easily be differentiated from all Lotus species by the absence of trichomes on the stem, leaf, and calyx.

\section{Group (III): Lotus polyphyllos, L. creticus and L. cytisoides}

Variations in the bract length (BL), upper leaflet length (ULL) and the length of calyx tube (CTU) were the significant quantitative continuous characters that differentiate among members of this group and between the others (Tab. 6). For quantitative cardinal character, between both species of Lotus, the shape of both upper (SUL) and lower (SLL) leaflets were of significant differences $(P=0.001)$. In the lower leaflets, from lanceolate to ovate and in the upper leaflets ranged between obovate to lanceolate. This group occupied the negative end along PCoA axis1, and overlapped with group (IV), and positioned in the centre of PCA ordination plane overlapping with groups (II) and (IV) without any correlations to other variables. With respect to micromorphological characters, Zareh et al. (2017) found a high similarity coefficient (0.75) between Lotus creticus and L. cytisoides as both have the same type of trichomes on the stem, leaf, and calyx as well as the same shape of seeds. In our study, the latter two species were closely related with each other forming a cluster together (Diag. 1) which supports Zareh et al. (2017) results.

\section{Group (IV): Lotus gebelia, L. lanuginosus and L. arenarius}

The members of this group can be differentiated among and between the others by variations in style length (STL, Tab. 6). The length of rachis (R) was the longest $(10 \mathrm{~mm})$ in Lotus gebalia, while it was the shortest in L. lanuginosus $(3 \mathrm{~mm})$. As a quantitative cardinal character, the style shape (STS) played a significant role in the morphological discrimination between this and other groups. In L. arenarius, it was bifid, while simple in the remaining two species. Lotus arenarius formed a separate branch in this cluster (Diag. 1). Differences in the ratio between seed and the pod (S/P) and the length of seeds (SL) shared the significant characters that helped in delimitation of species of groups (III) and (IV). Along the positive end of PCA axis 2, this group occupied the highest scores and showed a correlation to the style length (STL; Diag. 3).

\section{Group (V): Lotus edulis, L. tetragonolobus and L. conjugatus}

The discriminating significant $(P=0.001)$ quantitative continuous characters that can separate this group were the pod length (PL) and length of lower leaflet (LLL). It shared the significant variation in the number of ovules (NOV) with group (IV), and calyx length with group (VI). This can be illustrated in Diagrams (2) and (3) where this group occupied the highest positive scores along PCoA axis 1 and was affected by (PL), (NOV) and (LLL). Here, the variation in the shape of the pod (PWK) was significantly different and deliminates two 
species of his group: L. tetragonolobus with winged pod and L. conjugatus with a keeled pod. Along PCA axis 1, this group was spread at the negative end. The inclusion of $L$. tetragonolobus and L. conjugatus in this group is quite true and confirmed their taxonomic classification belonging to a separate Subgenus Tetragonolobus (Callen, 1959).

\section{Group (VI): Lotus ornithopodioides, L. peregrinus, L. arabicus and L. hebranicus}

Within the four species of this group, differences in seed color (SC) and shape of the upper leaflet (SUL) were the significant quantitative cardinal characters (Tab. 6). Lotus arabicus can be differentiated from the others by its green seed color, while the others have brown. The shape of upper leaflets varied from obovate to rhombic in $L$. ornithopodioides and $L$. peregrinus, and from obovate in L. arabicus and oblanceolate in L. hebranicus. The length of calyx teeth (CT) was the diagnostic character among the studied species. This fact becomes true when examining the PCA ordination diagram (Diag. 3). Together with a group (V), they positioned along the positive end of PCoA axis 1. The UPGMA dendrogram resulted from Zareh et al. (2017) placed L. arabicus and L. hebranicus as closely related branches in one cluster. In the current study, the latter two species separated into two close branches within the same cluster (Diag. 1).

\section{CONCLUSIONS}

Lotus is a taxonomically difficult genus. Using UPGMA clustering, PCoA and PCA analyses to both quantitative cardinal and continuous morphological vegetative and reproductive characters helped in the differentiation of the 19 species of Lotus. This study revealed the importance of pod length, seed dimensions, measurements of upper and lower leaflets, calyx, length of corolla, length of style, numbers of flowers and ovules as characteristics that discriminate between the studied taxa. Despite its being limited to some species of Lotus in Egypt, our results proposed diagnostic characters that were not previously used in the genus Lotus, and enabled the separation of six species-specific groups. Future more investigations and analyses using more characters to improve species delimitation are recommended. This becomes true especially to avoid overlapping of characters in closely related species.

\section{ACKNOWLEDGMENTS}

The authors would like to thank three anonymous reviewers for their revision and helpful comments on an earlier version of this manuscript.

\section{CONFLICT OF INTEREST STATMENT}

The authors have no conflicts of interest to declare.

\section{LITERATURE CITED}

Albert, S. and Sharma, B. 2013. Comparative foliar micromorphological studies of some Bauhinia (Leguminosae) species. Turkish Journal of Botany, 37: 276-281.

Allan, G. J. and Porter, J. M. 2000. Tribal delimitation and phylogenetic relationships of Loteae and Coronilleae (Faboideae: Fabaceae) with special reference to Lotus: 
Gaafar et al.

evidence from nuclear ribosomal ITS sequences. American Journal of Botany, 87(12):1871-1881.

Arambarri, A. M., Stenglein, S. A., Colares, M. N. and Novoa, M. C. 2005. Taxonomy of the New World species of Lotus (Leguminosae: Loteae). Australian Journal of Botany, 53: 797- 812 .

Ball, P. W. and Chrtková-Žertová, A. 1968. Lotus L. In: Tutin, T. G., Heywood, V. H., Burges, N. A., Moore, D. M., Valentine, S. M. and Webb, D. A. (eds.), Flora Europaea. Vol. 2: p. 173-176. Cambridge: Cambridge University Press.

Bateman, R. M. and Rudall, P. J. 2006. Evolutionary and morphometric implications of morphological variation among flowers within an inflorescence: A case-study using European orchids. Annals of Botany, 98(5): 975-993.

Boulos, L. 1999. Flora of Egypt. Azollaceae-Oxalidaceae, Vol 1. Al Hadara Publishing, Cairo, 419 pp.

Boulos, L. 2009. Flora of Egypt Checklist Revised Annotated Edition. Al-Hadara Publishing, Cairo, Egypt, 410 pp.

Brouillet, L. 2008. The taxonomy of North American Loti (Fabaceae: Loteae): new names in Acmispon and Hosackia. Journal of the Botanical Research Institute of Texas, 2: 387394.

Callen, E. O. 1959. Studies in the genus Lotus (Leguminosae). I. Limits and subdivisions of the genus. Canadian Journal of Botany, 37: 157-165.

Collenette, S. 1999. Wild flowers of Saudi Arabia. National Commission for Wildlife Conservation and Development, Riyadh, 799 pp.

Cortinhas, A., Erben, M., Paes, A. P., Santo, D. E., Guara-Requena, M. and Caperta, A. D. 2015. Taxonomic complexity in the halophyte Limonium vulgare and related taxa (Plumbaginaceae): insights from analysis of morphological, reproductive and karyological data. Annals of Botany, 115(3): 369-383.

Degtjareva, G., Kramina, T., Sokoloff, D., Samigullin, T., Valiejo-Roman, C. and Antonov, A. 2006. Phylogeny of the genus Lotus (Leguminosae, Loteae): evidence from nrITS sequences and morphology. Botany, 84 (5): 813-830.

El-Gazzar, A., Abd El-Ghani, M. M., El-Husseini, N. and Khattab, A. 2013. Classification of the Leguminosae-Papilionoideae: A Numerical re-assessment. Notulae Scientia Biologicae, 5(4): 499-507. 
El-Hadidi, M. N. 2000. The main features of the natural vegetation. In : El Hadidi, M. N. (eds.), Flora Aegyptiaca, Vol. 1, p.27- 105. The Palm Press, Cairo, Egypt.

El Hadidy, A. M. H. 2003. Systematic revision of Leguminosae in Egypt. 4. Lotus L. Taeckholmia, 23(1): 133-163.

El Hadidy, A. M. H. 2004. Morphological studies on fruits and seeds of the genus Lotus L. in Egypt. Proceedings of First International Conference on Strategy of Egyptian Herbaria, p. 129-159.

El-Hadidy, A., Abd El-Ghani, M. M., Amer, W. and Hassan, R. 2011. Systematic revision of the genus Pancratium L. (Amaryllidaceae) in Egypt with a new addition. Notulae Scientia Biologicae, 3(2): 24-38.

Ellmouni, F. Y., Karam, M. A., Ali R. M. and Albach, D. C. 2017. Molecular and morphometric analysis of Veronica L. section Beccabunga (Hill) Dumort. Aquatic Botany, 136: 95-111.

Faria, M., Harris, D. J., Necrasov, T. V., De Sousa, M. T. and Nunes, E. 2012. The correct phylogenetic position of Lotus conimbricensis Brot. (Leguminosae, Loteae) based on nuclear ribosomal ITS sequences. Acta Botanica Croatica, 71 (1): 87-94.

Gillett, J. B. 1958. Lotus in Africa south of the Sahara (excluding the Cape Verde islands and Socotra) and its distinction from Dorycnium. Kew Bulletin, 13(3): 361-381.

Gilmartin, A. J. 1967. Numerical Taxonomy - an eclectic viewpoint. Taxon, 16: 8-12.

Gower, J. C. 1971. Statistical methods of comparing different multivariate analyses of the same data. In: Hodson, F. R., Kendall, D. G. and Tautu, P. (eds.), Mathematics in the archaeological and historical sciences. Edinburgh University Press, Edinburgh and Aldine- Atherton, Chicago, p. 138-149.

Grant, W. F. 1991. Chromosomal evolution and aneuploidy in Lotus. In: Tsuchiga, T and Gupta, P. K. (ed). Chromosome engineering in plant, genetic breeding and evolution. Developments in plant genetics and breeding Elsevier, Amsterdam, The Netherlands, p. 493-529.

Hammer, Ø., Harper, D. A. and Ryan, P. D. 2001. PAST: paleontological statistics software package for education and data analysis. Palaeontologia Electronica, 4:1-9.

Heyn, C. C. 1967. The Lotus creticus group. Kew Bulletin, 21: 299-309.

Heyn, C. C. 1970. Studies in Lotus. III. The Lotus angustissimus group. Israel Journal of Botany, 19: 271-292. 
Gaafar et al.

Heyn, C. C. and Herrnstadt, I. 1968. The Lotus creticus group. Kew Bulletin, 21: 229-309.

Jensen, R. J. 2003. The conundrum of morphometrics. Taxon, 52: 663-671.

Jensen, R. J. and Eshbaugh, W. H. 1976. Numerical taxonomic studies of hybridization in Quercus. II. Populations with wide areal distribution and high taxonomic diversity. Systematic Botany, 1: 11-19.

Jensen, R. J., Hokanson, S. C., Isebrands, J. G. and Hancock, J. F. 1993. Morphometric variation in oaks of the Apostle Islands in Wiscons: evidence of hybridization between Quercus rubra and Q. ellipsoldalis (Fagaceae). American Journal of Botany, 80: 1358-1366.

Kramina, T. E. 1999. A contribution to the taxonomic revision of the Lotus corniculatus complex (Leguminosae, Loteae) in the European part of the former USSR. Systematics and Geography of Plants, 68: 265-279.

Kramina, T. E. 2006. A contribution to the taxonomic revision of the Lotus angustissimuscomplex (Leguminosae, Loteae). Wulfenia, 13: 57-92.

Kramina, T. E. and Sokoloff, D. D. 2003. On Lotus Section Erythrolotus and related taxa (Leguminosae). Bulletin of Moscow Society of Naturalists. Biological series, 108: 59-62. [In Russian].

Kramina, T. E. and Sokoloff, D. D. 2004. A taxonomic study of Lotus australis complex (Leguminosae), with special emphasis on plants from Pacific Ocean Islands. Adansonia, Ser. 3, 26(2): 171-197.

Kramina, T. E., Degtjareva, G. V., Samigullin, T. H., Valiejo-Roman, C. M., Kirkbride, J. H. Jr., Volis, S., Tao, D. and Sokoloff D. D. 2016. Phylogeny of Lotus (Leguminosae: Loteae): partial incongruence between nrITS, nrETS and plastid markers and biogeographic implications. Taxon, 65(5): 997-1018.

Kramina, T. E., Lysova, M., Samigullin, T., Schanzer, I., Özbek‘ M. and Sokoloff, D. 2021. Phylogenetic placement and phylogeography of large-flowered Lotus species (Leguminosae) formerly classified in Dorycnium: Evidence of pre-pleistocene differentiation of western and eastern intraspecific groups. Plants, 10 (2): 260.

Kramina, T. E., Meshersky, I. G., Degtjareva, G. V., Samigullin, T. H., Belokon, Y.S. and Schanzer, I. A. 2018. Genetic variation in the Lotus corniculatus complex (Fabaceae) in Northern Eurasia as inferred from nuclear microsatellites and plastid trnL-trnF sequences. Botanical Journal of the Linnean Society, 188: 87-116. 
Kramina, T. E., Samigullin, T. H. and Meschersky, I. G. 2020. Two cryptic species of Lotus (Fabaceae) from the Iberian Peninsula. Wulfenia, 27: 21-45.

Legendre, P. and Legendre, L. 1998. Numerical ecology, 2nd English edn. Elsevier, Amsterdam, $852 \mathrm{pp}$.

Macleod, N. and Fore, P. L. (eds.) 2002. Morphology, shape and phylogeny. The Systematics Association Special Volume Series, 64. Taylor \& Francis, London, 308 pp.

McNeil, J. 1984. Numerical taxonomy and biosystematics. In: Grant, W. F. (ed.), Plant Biosystematics. Academic Press, Toronto, 690 pp.

Muschler, R. 1912. A Manual flora of Egypt. Vol. 2, Berlin, 1312 pp.

Ojeda, I., Francisco-Ortega, J. and Cronk, Q. C. 2009. Evolution of petals epidermal micromorpholgy in Leguminosae and its use as a marker of petal identity. Annals of Botany, 104: 1099-1110.

Saheed, S. A. and Illoh, H. C. 2010. A taxonomic study of some species in Cassiinae (Leguminosae) using leaf epidermal characters. Notulae Botanicae Horti Agrobotanici Cluj-Napoca, 38: 21-27.

Sneath, P. H. A. and Sokal, R. R. 1973. Numerical taxonomy: the principles and practice of numerical classification. Freeman W. H., San Francisco, 573 pp.

Sokal, R. R. 1986. Phenetic taxonomy: theory and methods. The Annual Review of Ecology, Evolution, and Systematics, 17: 423-442.

Sokal, R. R. and Rohlf, F. J. 1981. Biometry: the principles and practice of statistics in biological research (second edition): San Francisco: W. H. Freeman and Company, 859 pp.

Sokal, R. R. and Sneath, P. H. A. 1963. Principles of numerical taxonomy. W. H. Freeman and Company, San Francisco, 359 pp.

Sokoloff, D. D. 1998. Morphlogical and taxonomical study of the genus Anthyllis and principles of revision of Loteae tribe (Papilionaceae). Ph.D. dissertation. Moscow University, Moscow, Russia. (In Russian).

Sokoloff, D. D. 1999a. Ottleya, a new genus of Papilionaceae-Loteae from North America. Feddes Repertorium, 110: 89-97.

Sokoloff, D. D. 1999b. Quid est Tetragonolobus wiedemannii Boiss. (Fabaceae)? Novitates Systematicae Plantarum Vascularium (St. Petersburg), 31: 139-142. (In Russian). 
Gaafar et al.

Sokoloff, D. D. and Lock, J. M. 2005. Tribe Loteae. In: Lewis, G., Schrire, B., Mackinder, B. and Lock, M. (eds.), Legumes of the world. Royal Botanic Gardens, Kew, UK. p. 455465 .

Sokoloff, D. D., Degtjareva, G. V., Endress, P. K., Remizowa, M. V., Samigullin, T. H. and Valiejo-Roman, C. M. 2007. Inflorescence and early flower development in Loteae (Leguminosae) in a phylogenetic and taxonomic context. International Journal of Plant Sciences, 168(6): 801-833.

Španiel, S., Zozomová-Lihová, J. and Marhold, K. 2017. Revised taxonomic treatment of the Alyssum montanum - A. repens complex in the Balkans: A multivariate morphometric analysis. Plant Systematics and Evolution, 303: 1413-1442.

Stenglein, S. A., Colares, M. N., Arambarri, A. M., Novoa, M. C., Vizcaino, C. E. and Katinas, L. 2003. Leaf epidermal microcharacters of the Old World species of Lotus (Leguminosae: Loteae) and their systematic significance. Australian Journal of Botany, 51: 459-469.

Täckholm, V. 1974. Students' Flora of Egypt. 2nd Edition. Cairo University Press, Cairo, Egypt, 888 pp.

Ter Braak, C. J. F. and Šmilauer, P. 2003. CANOCO reference manual and user's guide to Canoco for Windows: software for canonical community ordination (version 4.5). Ithaca, NY: Microcomputer Power.

Thiers, B. 2017. Index herbariorum: a global directory of public herbaria and associated staff. Retrieved Jul. 2, 2018. Available from http://sweetgum.nybg.org.

Weiß, C. H. 2007. StatSoft, Inc., Tulsa, OK.: STATISTICA, Version 8. AStA Advances in Statistical Analysis, 91: 339-341.

Wiens, J. J. 2000. Phylogenetic analysis of morphological data. Smithsonian Institution Press, Comparative Evolutionary Biology Series, 232 pp.

Zareh, M., Faried, A. and Farghaly, N. 2017. Micromorphological studies on the genus Lotus L. (Fabaceae: Loteae) from Egypt. Turkish Journal of Botany, 41: 273-288.

Zohary, M. 1972. Flora Palaestina. Part 2, Text Platanaceae to Umbelliferae. Israel Academy of Science and Humanities, Jerusalem, 656 pp.

Zorić, L., Metkulov, L., Lukovic, J., Boza, P. and Polic, D. 2009. Leaf epidermal characteristics of Trifolium L. species from Serbia and Montenegro. Flora, 204: 198209. 


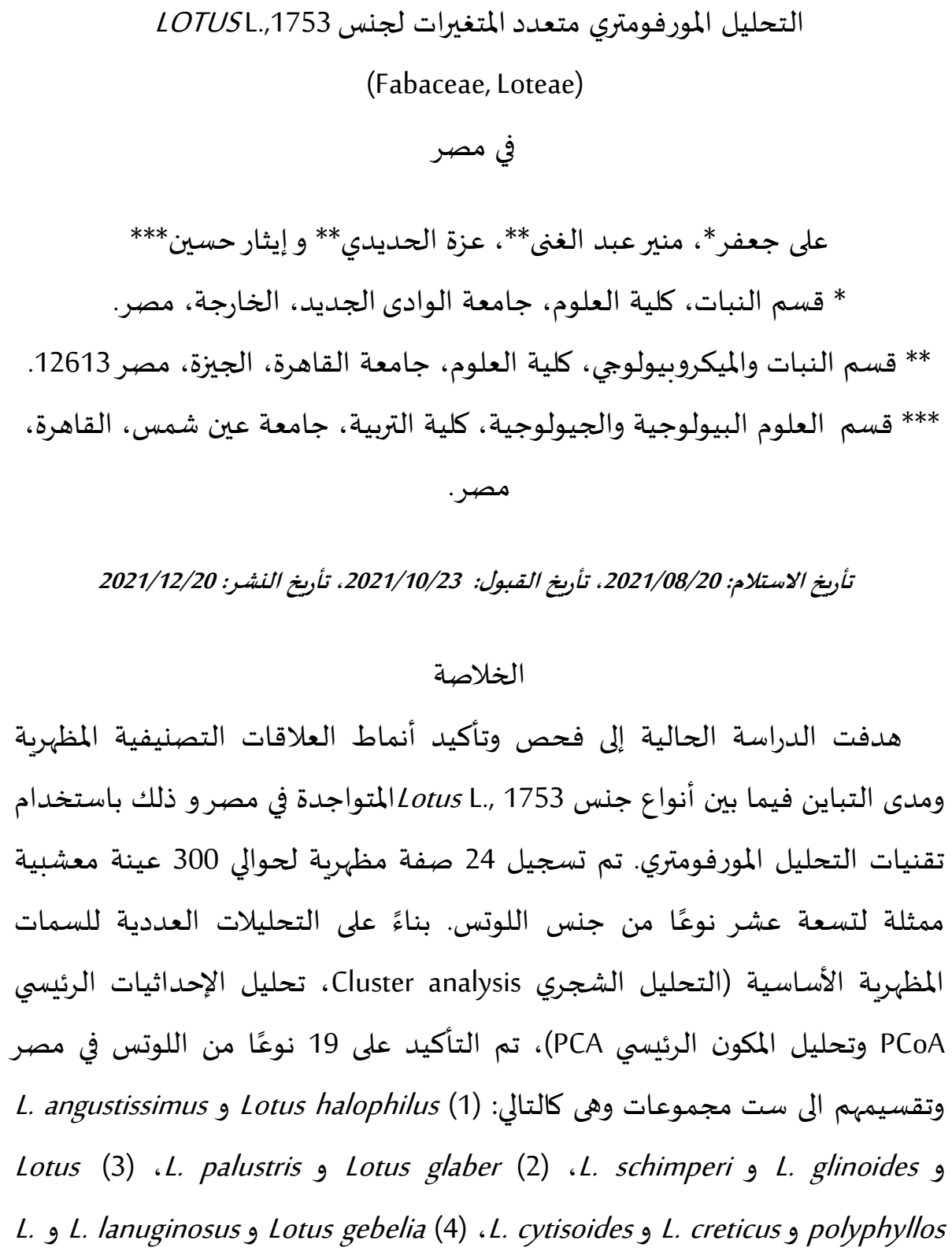
Lotus (3) ، L. palustris و Lotus glaber (2) ،L. schimperi و L. glinoides و L. و L L lanuginosus و Lotus gebelia (4) ، L. cytisoides و L. creticus و polyphyllos 
Gaafar et al.

Lotus (6) ، L. conjugatus و L. tetragonolobus و Lotus edulis (5) ،arenarius .L. hebranicus و L. arabicus و L.peregrinus g ornithopodioides

أظهرت النتائج ان بعض الصفات- التي تم فحصها للمرة الأولى في هذا البحث- كان

لها تأثير معنوي في فصل الأنواع المختلفة مثل طول القرن، أبعاد البذور، سمات

الوريقات العلوية والسفلية، طول كلا من الكأس والتويج والقلم، بالإضافة الى عدد

الأزهاروالبويضات. 